Cochrane Database of Systematic Reviews

\title{
Routine monitoring of gastric residual for prevention of necrotising enterocolitis in preterm infants (Review)
}

Abiramalatha T, Thanigainathan S, Ninan B

Abiramalatha T, Thanigainathan S, Ninan B.

Routine monitoring of gastric residual for prevention of necrotising enterocolitis in preterm infants.

Cochrane Database of Systematic Reviews 2019, Issue 7. Art. No.: CD012937.

DOI: 10.1002/14651858.CD012937.pub2.

www.cochranelibrary.com 
TABLE OF CONTENTS

HEADER

ABSTRACT

PLAIN LANGUAGE SUMMARY

SUMMARY OF FINDINGS

BACKGROUND

OBJECTIVES

METHODS

RESULTS

Figure 1.

Figure 2.

Figure 3.

Figure 4.

Figure 5.

Figure 6.

Figure 7.

DISCUSSION

AUTHORS' CONCLUSIONS

ACKNOWLEDGEMENTS

REFERENCES

CHARACTERISTICS OF STUDIES

DATA AND ANALYSES

Analysis 1.1. Comparison 1 Routine monitoring of gastric residuals versus no monitoring of gastric residuals, Outcome 1 Number of infants with NEC stage 2 or 3.

Analysis 1.2. Comparison 1 Routine monitoring of gastric residuals versus no monitoring of gastric residuals, Outcome 2 Time to reach full enteral feeds.

Analysis 1.3. Comparison 1 Routine monitoring of gastric residuals versus no monitoring of gastric residuals, Outcome 3 Number of infants with surgical NEC.

Analysis 1.4. Comparison 1 Routine monitoring of gastric residuals versus no monitoring of gastric residuals, Outcome 4 Time to regain birth weight (days).

Analysis 1.5. Comparison 1 Routine monitoring of gastric residuals versus no monitoring of gastric residuals, Outcome 5 Number of infants with extrauterine growth restriction at discharge.

Analysis 1.6. Comparison 1 Routine monitoring of gastric residuals versus no monitoring of gastric residuals, Outcome 6 Number of infants with episodes of interruption of feeds (lasting $\geq 12$ hours).

Analysis 1.7. Comparison 1 Routine monitoring of gastric residuals versus no monitoring of gastric residuals, Outcome 7 Number of TPN days.

Analysis 1.8. Comparison 1 Routine monitoring of gastric residuals versus no monitoring of gastric residuals, Outcome 8 Number of infants with parenteral nutrition-associated liver disease.

Analysis 1.9. Comparison 1 Routine monitoring of gastric residuals versus no monitoring of gastric residuals, Outcome 9 Number of days of CVL usage.

Analysis 1.10. Comparison 1 Routine monitoring of gastric residuals versus no monitoring of gastric residuals, Outcome 10 Number of infants with invasive infection.

Analysis 1.11. Comparison 1 Routine monitoring of gastric residuals versus no monitoring of gastric residuals, Outcome 11 Allcause mortality before discharge.

Analysis 1.12. Comparison 1 Routine monitoring of gastric residuals versus no monitoring of gastric residuals, Outcome 12 Duration of hospital stay (days).

Analysis 2.1. Comparison 2 Using two different criteria of gastric residual for feed interruption while monitoring gastric residual, Outcome 1 Number of infants with NEC stage 2 or 3.

Analysis 2.2. Comparison 2 Using two different criteria of gastric residual for feed interruption while monitoring gastric residual, Outcome 2 TIme to reach full enteral feeds.

Analysis 2.3. Comparison 2 Using two different criteria of gastric residual for feed interruption while monitoring gastric residual, Outcome 3 Number of infants with surgical NEC.

Analysis 2.4. Comparison 2 Using two different criteria of gastric residual for feed interruption while monitoring gastric residual, Outcome 4 Time to regain birth weight (days). 
Analysis 2.5. Comparison 2 Using two different criteria of gastric residual for feed interruption while monitoring gastric residual, Outcome 5 Number of infants with extrauterine growth restriction at discharge.

Analysis 2.6. Comparison 2 Using two different criteria of gastric residual for feed interruption while monitoring gastric residual, Outcome 6 Number of infants with episodes of interruption of feeds (lasting $\geq 12$ hours).

Analysis 2.7. Comparison 2 Using two different criteria of gastric residual for feed interruption while monitoring gastric residual, Outcome 7 Number of TPN days.

Analysis 2.8. Comparison 2 Using two different criteria of gastric residual for feed interruption while monitoring gastric residual, Outcome 8 Number of infants with Invasive Infection.

Analysis 2.9. Comparison 2 Using two different criteria of gastric residual for feed interruption while monitoring gastric residual, Outcome 9 All-cause mortality before discharge. 
[Intervention Review]

\section{Routine monitoring of gastric residual for prevention of necrotising enterocolitis in preterm infants}

Thangaraj Abiramalatha ${ }^{1}$, Sivam Thanigainathan ${ }^{2}$, Binu Ninan ${ }^{1}$

1Neonatology, Sri Ramachandra Institute of Higher Education and Research, Chennai, India. 2Neonatology, Jawaharlal Institute of Postgraduate Medical Education and Research Puducherry, Puducherry, India

Contact address: Thangaraj Abiramalatha, Neonatology, Sri Ramachandra Institute of Higher Education and Research, Chennai, Tamil Nadu, India.abi_paeds@yahoo.com.

Editorial group: Cochrane Neonatal Group.

Publication status and date: New, published in Issue 7, 2019.

Citation: Abiramalatha T, Thanigainathan S, Ninan B. Routine monitoring of gastric residual for prevention of necrotising enterocolitis in preterm infants. Cochrane Database of Systematic Reviews 2019, Issue 7. Art. No.: CD012937. DOI: 10.1002/14651858.CD012937.pub2.

Copyright @ 2019 The Cochrane Collaboration. Published by John Wiley \& Sons, Ltd.

\section{A B S T R A C T}

\section{Background}

Routine monitoring of gastric residual in preterm infants on gavage feeds is a common practice that is used to guide initiation and advancement of feeds. Some literature suggests that an increase in/or an altered gastric residual may be predictive of necrotising enterocolitis. Withholding monitoring of gastric residual may take away the early indicator and thus may increase the risk of necrotising enterocolitis. However, routine monitoring of gastric residual as a guide, in the absence of uniform standards, may lead to unnecessary delay in initiation and advancement of feeds and delay in reaching full enteral feeds. This in turn may increase the duration of parenteral nutrition and central venous line usage, increasing their complications. Delay in achieving full enteral feeds increases the risk of extrauterine growth restriction and neurodevelopmental impairment.

\section{Objectives}

- To assess the efficacy and safety of routine monitoring of gastric residual versus no monitoring of gastric residual in preterm infants

- To assess the efficacy and safety of routine monitoring of gastric residual based on two different criteria for interrupting feeds or decreasing feed volume in preterm infants

We planned to undertake subgroup analysis based on gestational age ( $\leq 27$ weeks, 28 weeks to 31 weeks, $\geq 32$ weeks), birth weight ( $<1000$ $\mathrm{g}, 1000 \mathrm{~g}$ to $1499 \mathrm{~g}, \geq 1500 \mathrm{~g}$ ), small for gestational age versus appropriate for gestational age infants (classified using birth weight relative to the reference population), type of feed the infant is receiving (human milk or formula milk), and frequency of monitoring of gastric residual (before every feed, before every third feed, etc.) (see "Subgroup analysis and investigation of heterogeneity").

\section{Search methods}

We used the standard search strategy of Cochrane Neonatal to search the Cochrane Central Register of Controlled Trials (CENTRAL; 2018, Issue 1), MEDLINE via PubMed (1966 to 19 February 2018), Embase (1980 to 19 February 2018), and the Cumulative Index to Nursing and Allied Health Literature (CINAHL; 1982 to 19 February 2018). We also searched clinical trials databases, conference proceedings, and the reference lists of retrieved articles for randomised controlled trials and quasi-randomised trials.

\section{Selection criteria}

We selected randomised and quasi-randomised controlled trials that compared routine monitoring of gastric residual versus no monitoring or two different criteria of gastric residual to interrupt feeds in preterm infants. 


\section{Data collection and analysis}

Two review authors assessed trial eligibility and risk of bias and independently extracted data. We analysed treatment effects in individual trials and reported the risk ratio and the risk difference for dichotomous data, and the mean difference for continuous data, with respective $95 \%$ confidence intervals. We used the GRADE approach to assess the quality of evidence.

\section{Main results}

Two randomised controlled trials with a total of 141 preterm infants met the inclusion criteria for the comparison of routine monitoring versus no monitoring of gastric residual in preterm infants. Both trials were done in infants with birth weight $<1500 \mathrm{~g}$.

Routine monitoring of gastric residual may have little or no effect on the incidence of necrotising enterocolitis (risk ratio (RR) 3.07, 95\% confidence interval ( $\mathrm{Cl}$ ) 0.50 to 18.77 ; participants $=141$; studies $=2$; low-quality evidence). Routine monitoring may increase the risk of feed interruption episodes ( $\mathrm{RR} 2.07,95 \% \mathrm{Cl} 1.39$ to 3.07; participants = 141; studies = 2; low-quality evidence); the number needed to treat for an additional harmful outcome (NNTH) was 3 (95\% $\mathrm{Cl} 2$ to 6$)$.

Routine monitoring of gastric residual may increase time taken to establish full enteral feeds (mean difference (MD) $3.92,95 \% \mathrm{Cl} 2.06$ to 5.77 days; participants $=141$; studies $=2$; low-quality evidence), time taken to regain birth weight (MD $1.70,95 \% \mathrm{Cl} 0.01$ to 3.39 days; participants $=80$; studies $=1$; low-quality evidence), and number of total parenteral nutrition days (MD 3.29, $95 \% \mathrm{Cl} 1.66$ to 4.92 days; participants = 141 ; studies $=2$; low-quality evidence).

We are uncertain as to the effect of routine monitoring of gastric residual on other outcomes such as incidence of surgical necrotising enterocolitis, extrauterine growth restriction at discharge, parenteral nutrition-associated liver disease, duration of central venous line (CVL) usage, incidence of invasive infection, mortality before discharge, and duration of hospital stay. We found no data for outcomes such as aspiration pneumonia, gastroesophageal reflux, growth measures following discharge, and neurodevelopmental outcome.

Only one trial with 87 preterm infants met the inclusion criteria for the comparison of using two different criteria of gastric residual to interrupt feeds while monitoring gastric residual. The trial was done in infants with birth weight of 1500 to $2000 \mathrm{~g}$. We are uncertain as to the effect of using two different criteria of gastric residual on outcomes such as incidence of necrotising enterocolitis or surgical necrotising enterocolitis, time to establish full enteral feeds, time to regain birth weight, number of total parenteral nutrition days, number of infants experiencing feed interruption episodes, extrauterine growth restriction at discharge, parenteral nutrition-associated liver disease, incidence of invasive infection, and mortality before discharge (very low quality evidence). We found no data on duration of CVL usage, aspiration pneumonia, gastroesophageal reflux, duration of hospital stay, growth measures following discharge, and neurodevelopmental outcome.

\section{Authors' conclusions}

Review authors found insufficient evidence as to whether routine monitoring of gastric residual reduces the incidence of necrotising enterocolitis because trial results are imprecise. Low-quality evidence suggests that routine monitoring of gastric residual increases the risk of feed interruption episodes, increases the time taken to reach full enteral feeds and to regain birth weight, and increases the number of total parenteral nutrition (TPN) days.

Available data are insufficient to comment on other major outcomes such as incidence of invasive infection, parenteral nutrition-associated liver disease, mortality before discharge, extrauterine growth restriction at discharge, number of CVL days, and duration of hospital stay. Further randomised controlled trials are warranted to provide more precise estimates of the effects of routine monitoring of gastric residual on important outcomes, especially necrotising enterocolitis, in preterm infants.

\section{PLAIN LANGUAGE SUMMARY}

\section{Routine monitoring of stomach aspirates for prevention of necrotising enterocolitis in preterm infants}

\section{Review question}

Does routine monitoring of stomach aspirates in preterm infants prevent necrotising enterocolitis?

\section{Background}

Monitoring of stomach aspirates to diagnose feed intolerance and necrotising enterocolitis is a common practice in preterm infants who are on tube feeds. No adequate evidence supports routine monitoring of stomach aspirates as a guide to initiating and increasing feeds in otherwise healthy preterm infants. Withholding monitoring of stomach aspirates may take away the early indicator and thus may increase the risk of necrotising enterocolitis. However, routine monitoring of stomach aspirates as a guide, in the absence of uniform standards, may lead to unnecessary delay in initiation and advancement of feeds and delay in reaching full feeds. This in turn may increase the duration of parenteral nutrition and central line usage, thus increasing their complications. Delay in achieving full feeds increases the risk of growth restriction and neurodevelopmental impairment. We have looked for evidence from clinical trials that assessed whether routine monitoring of stomach aspirates is beneficial or harmful in preterm infants.

\section{Study characteristics}


The literature searches are up-to-date as of 19 February 2018. We found two small randomised controlled trials with a total of 141 preterm infant participants that compared routine monitoring versus no monitoring of stomach aspirates in preterm infants. We found one trial for the comparison of using two different criteria of aspirates to interrupt feeds while monitoring stomach aspirates in preterm infants.

\section{Key results}

There is uncertainty as to whether routine monitoring of stomach aspirates reduces necrotising enterocolitis because trial results are imprecise. Preterm infants on routine monitoring of stomach aspirates may reach full feeds later, regain birth weight later, require longer duration of parenteral nutrition, and may be at increased risk of feed interruption episodes.

There is uncertainty as to whether using two different criteria of gastric residual to interrupt feeds has effect on important outcomes in preterm infants.

\section{Conclusions}

There is uncertainty as to whether routine monitoring of stomach aspirates has any benefits. Routine monitoring may increase the number of feed interruption episodes and the time taken to reach full feeds. 


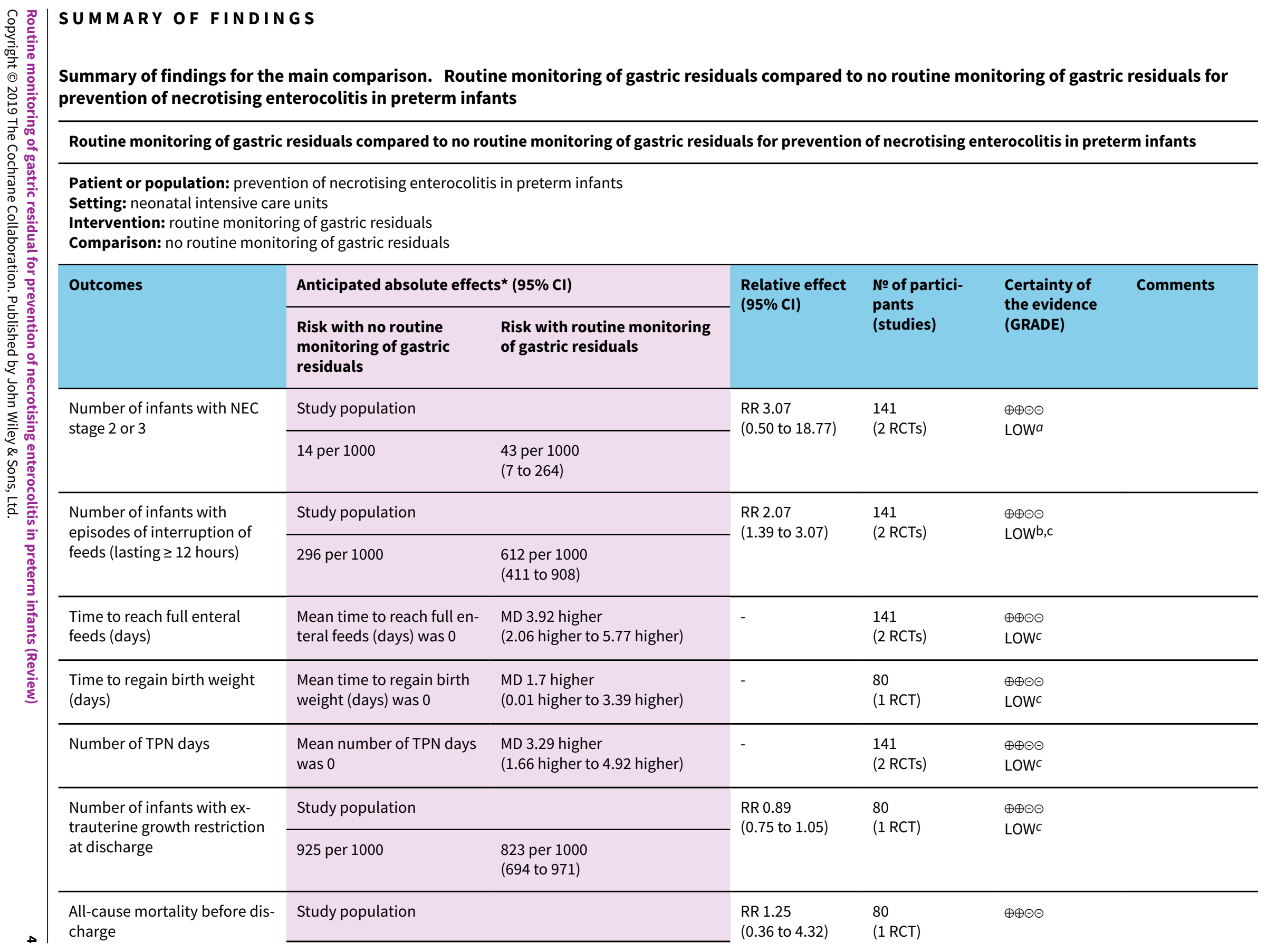


* The risk in the intervention group (and its $95 \%$ confidence interval) is based on the assumed risk in the comparison group and the relative effect of the intervention (and its $95 \% \mathrm{Cl})$.

Cl: confidence interval; MD: mean difference; RCT: randomised controlled trial; RR: risk ratio.

\section{GRADE Working Group grades of evidence.}

High certainty: we are very confident that the true effect lies close to that of the estimate of the effect.

Moderate certainty: we are moderately confident in the effect estimate: the true effect is likely to be close to the estimate of the effect, but there is a possibility that it is substantially different.

Low certainty: our confidence in the effect estimate is limited: the true effect may be substantially different from the estimate of the effect.

Very low certainty: we have very little confidence in the effect estimate: the true effect is likely to be substantially different from the estimate of effect.

almprecise due to small sample size and low event rate.

bPossibility of bias due to lack of blinding.

cImprecise due to small sample size. 


\section{B A C K G R O U N D}

\section{Description of the condition}

Providing adequate nutrition is a key component of the healthcare of preterm neonates. There is increasing emphasis on early initiation and appropriate advancement of enteral feeds with the aim of achieving full-volume enteral feeds at the earliest (Dutta 2015; Stevens 2016). Major hindrances to advancing feed volumes in preterm infants include feed intolerance and the risk of necrotising enterocolitis (NEC).

Feed intolerance is a common problem in preterm infants and is due to structural and functional immaturity of the gut of preterm infants. The preterm gut has decreased length, immature motility patterns, and inadequate digestive and absorptive capacity compared to the gut of term infants (Lucchini 2011). Feed intolerance causes frequent interruption and delayed advancement of enteral feeds, resulting in protracted use of total parenteral nutrition (TPN) and central venous lines (CVLS), increasing their complication rates (Hermansen 2005; Duro 2011; Kaur 2015). Delay in establishing full enteral feeds is a significant contributor to growth failure in preterm infants, resulting in neurodevelopmental impairment and long-term metabolic complications (Franz 2009; Embleton 2013; Stevens 2016).

\section{Description of the intervention}

Feed intolerance is variously defined by signs such as increased volume of gastric residual, altered gastric residual (bilious- or blood-stained), abdominal distension, and/or emesis (Moore 2011). The use of gastric residual as an indicator of feed intolerance is controversial (Li 2014; Parker 2015).

Gastric residual is the measure of the volume of milk along with gastrointestinal secretions remaining in the stomach after a certain time interval. Increased gastric residual is common in preterm infants due to the inherent immaturity of the gastrointestinal system in the form of delayed gastric emptying, slower intestinal transit, inadequate secretion of gut hormones and enzymes, and possible duodeno-gastric reflux (Ittmann 1992; Riezzo 2000). Apart from these intrinsic factors, some extrinsic factors such as formula feeds; certain drugs such as theophyllines, mydriatics, and opioids; body position; and sickness of the baby further delay gastric emptying, thus contributing to increased gastric residual (Malhotra 1992; Cohen 2004; Li 2014).

Routine monitoring of gastric residual (volume and/or colour) in preterm infants on gavage feeds is a common practice in many neonatal intensive care units (NICUs) and is used to guide advancement of gavage feeds (Gregory 2012). An increase in or altered gastric residual is putatively considered a sign of feed intolerance or an early sign of NEC (Li 2014). Gastric residual becomes important when accompanied by other signs such as bilious vomiting, decreased bowel sounds, abdominal distension, abdominal wall erythema (redness of the skin), gross or occult blood in the stool, apnoea, bradycardia, and temperature instability. The significance of increased gastric residual as an isolated finding is uncertain.

The volume and/or colour of the gastric residual that definitively indicates feed intolerance, or is predictive of NEC, is unclear (Malhotra 1992; Cobb 2004; Kenton 2004; Bertino 2009; Dutta 2015; Parker 2015; Gephart 2017). As a consequence, there is wide variation in practice across NICUs. The various cut-offs used to define significant volume of gastric residual are $\geq 2 \mathrm{~mL} / \mathrm{kg}$ of the infant's weight, $>2 \mathrm{~mL}$ or $3 \mathrm{~mL}$ depending on the infant's weight, $>30 \%$ of the previous feed volume, and $>50 \%$ of the cumulative feed volume given during the time interval (Mihatsch 2002; Kaur 2015; Torrazza 2015; Grino 2016). Similarly, there is no standard recommendation for the frequency of assessment of gastric residual.

Increase in abdominal girth is the other commonly used sign of feed intolerance. An increase in abdominal girth greater than or equal to $2 \mathrm{~cm}$ is considered significant (Malhotra 1992; Lucchini 2011; Kaur 2015). However, measurement of abdominal girth is highly prone to interobserver and intraobserver variability. Evidence is inadequate to indicate that abdominal girth is a reliable measure of feed tolerance (Dutta 2015).

\section{How the intervention might work}

Some literature suggests that an increase in/or an altered gastric residual may be predictive of NEC (Cobb 2004; Bertino 2009; Grino 2016). Withholding monitoring of gastric residual may take away the early indicator and thus may increase the risk of NEC and its complications, including mortality. Without aspiration at regular intervals, the gastric residual may accumulate in the stomach and cause gastric distension, increasing the risk of gastroesophageal reflux and aspiration pneumonia.

The use of gastric residual as a guide in the absence of uniform standards on its use may lead to unnecessary delay in initiation and advancement of feeds or interruption of feeds in preterm infants (Shulman 2011; Kaur 2015). This may result in delay in reaching full enteral feeds, which in turn may increase the duration of TPN and the risk of parenteral nutrition-associated liver disease (Duro 2011; Kaur 2015). It may also increase the number of days of CVL usage, thus increasing the risk of late-onset sepsis and other CVL-related complications (Hermansen 2005). Delay in achieving full enteral feeds increases the risk of extrauterine growth restriction and neurodevelopmental impairment (Morris 1999; Franz 2009; Leppänen 2014). The negative pressure created by repeated aspirations, especially when the tip of the nasogastric (NG)/orogastric (OG) tube remains in close contact with the gastric mucosa, has the potential to damage the gastric mucosa (Li 2014). Moreoever, the volume of aspirated gastric residual may not be a reliable and accurate measure of residual gastric content, and it varies with baby's position, size of the nasogastric tube, aspiration technique, and viscosity of feeds (Bartlett 2015; Parker 2015).

Other major confusion surrounds whether to discard or re-feed the aspirated gastric residual (Juvé-Udina 2009; Williams 2010; Dutta 2015). This question is addressed in another Cochrane Review (Abiramalatha 2018).

The gastric residual contains milk, gastrointestinal enzymes, and hormones that aid in digestion and promote gastrointestinal motility and maturation; discarding this may have a negative influence on the infant's gastrointestinal system.

\section{Why it is important to do this review}

Given the potential use of gastric residual as an early indicator of NEC, as well as the possible risks of its routine monitoring, we undertook a systematic review to identify and appraise data from randomised controlled trials to provide a synthesis of evidence 
to inform practice and research. We found no existing systematic review of this topic.

\section{O B JE C T IVES}

- To assess the efficacy and safety of routine monitoring of gastric residual versus no monitoring of gastric residual in preterm infants

- To assess the efficacy and safety of routine monitoring of gastric residual based on two different criteria for interrupting feeds or decreasing feed volume in preterm infants

We planned to undertake subgroup analysis based on gestational age ( $\leq 27$ weeks, 28 weeks to 31 weeks, $\geq 32$ weeks), birth weight (<1000 g, $1000 \mathrm{~g}$ to $1499 \mathrm{~g}, \geq 1500 \mathrm{~g}$ ), small for gestational age versus appropriate for gestational age infants (classified using birth weight relative to the reference population), type of feed the infant is receiving (human milk or formula milk), and frequency of monitoring of gastric residual (before every feed, before every third feed, etc.) (Subgroup analysis and investigation of heterogeneity).

\section{METHOD S}

\section{Criteria for considering studies for this review}

\section{Types of studies}

Randomised or quasi-randomised trials and cluster-randomised trials were eligible for inclusion in the review.

\section{Types of participants}

Preterm ( $<37$ weeks' gestation) infants who did not have any overt sign of feed intolerance/NEC such as bilious vomiting, decreased bowel sounds, abdominal distension, abdominal wall erythema, gross or occult blood in the stool, apnoea, bradycardia, or temperature instability.

The infant should be on gavage feeds (nasogastric (NG) tube). Randomisation should have been done at the time of initiation of enteral feeds. Babies on respiratory support were also eligible, if they did not have any sign of feed intolerance/NEC.

\section{Types of interventions}

\section{Comparison 1}

\section{Intervention}

Routine monitoring of gastric residual to decide advancement of enteral feeds in infants who did not have any sign of feed intolerance/NEC. Gastric residual monitoring could be done at any time interval (e.g. before every feed, before every third feed) at the investigator's discretion.

Note: The investigator could have had predefined criteria for the quantity and quality of gastric residual to decide feed interruption or to decrease the feed volume.

\section{Control}

No monitoring of gastric residual in otherwise healthy babies until any sign of feed intolerance/NEC appeared. The control group could receive 'no monitoring for any sign of feed intolerance' or 'routine monitoring of other signs of feed intolerance such as increase in abdominal girth'.

\section{Comparison 2}

Monitoring of gastric residual was done in both intervention and control groups, and the decision on feeds (advancement/ continuation/decrease/interruption) was based on two different predefined criteria of gastric residual. The criteria for gastric residual could be based on its quality and/or quantity.

\section{Types of outcome measures}

\section{Primary outcomes}

- Number of infants with necrotising enterocolitis (NEC) stage 2 or 3 (modified Bell's staging; Walsh 1986)

- Time to establish full enteral feeds $\geq 150 \mathrm{~mL} / \mathrm{kg} / \mathrm{d}$ (days)

\section{Secondary outcomes}

- Number of infants with surgical NEC

- Time to regain birth weight (days) and subsequent rate of weight gain $(\mathrm{g} / \mathrm{kg} / \mathrm{d})$, linear growth $(\mathrm{cm} /$ week), and increase in head circumference $(\mathrm{cm} /$ week) during the initial hospitalisation period

- Number of infants with extrauterine growth restriction at discharge (number of infants who remain below the 10th percentile for the index population for weight, length, and head circumference)

- Number of episodes of interruption of feeds (lasting $\geq 12$ hours)

- Number of days of total parenteral nutrition (TPN)

- Number of infants with parenteral nutrition-associated liver disease

- Number of days of central venous line (CVL) usage

- Incidence of invasive infection as determined by culture of bacteria or fungus from blood, cerebrospinal fluid, or urine, or from a normally sterile body space

- Number of infants with aspiration pneumonia or pneumonitis (clinical or radiological evidence of lower respiratory tract compromise that has been attributed to covert or evident aspiration of gastric contents)

- Number of infants with gastroesophageal reflux diagnosed by clinical features - postfeed (if bolus-fed) apnoea, desaturation, irritability, vomiting; or oesophageal $\mathrm{pH}$ monitoring, multiple intraluminal impedance, or endoscopy

- All-cause mortality before discharge or up to 44 weeks' postmenstrual age

- Duration of hospital stay (days)

- Growth measures following discharge from hospital to latest follow-up (weight, length, and head circumference)

- Neurodevelopmental outcomes assessed after 12 months' corrected age: neurological evaluations; developmental scores; and classifications of disability, including auditory and visual disability. We will define neurodevelopmental impairment as the presence of one or more of the following: non-ambulant cerebral palsy; developmental quotient more than two standard deviations below the population mean; and blindness (visual acuity $<6 / 60$ ) or deafness (any hearing impairment requiring - or unimproved by - amplification) 


\section{Search methods for identification of studies}

We used the criteria and standard methods of Cochrane and Cochrane Neonatal (see the Cochrane Neonatal search strategy for specialized register).

\section{Electronic searches}

We conducted a comprehensive search including the Cochrane Central Register of Controlled Trials (CENTRAL; 2018, Issue 1) in the Cochrane Library; MEDLINE via PubMed (1996 to 19 February 2018); Embase (1980 to 19 February 2018); and the Cumulative Index to Nursing and Allied Health Literature (CINAHL; 1982 to 19 February 2018) using the following search terms: (gastric residual* OR aspirate ${ }^{\star}$ ), plus database-specific limiters for randomised controlled trials (RCTs) and neonates (see Appendix 1 for the full search strategies for each database). We did not apply language restrictions

We searched clinical trials registries (clinicaltrials.gov; the World Health Organization's International Trials Registry and Platform, and the ISRCTN Registry) for ongoing or recently completed trials.

\section{Searching other resources}

We also searched the reference lists of any articles selected for inclusion in this review to identify additional relevant articles. We searched the proceedings of the annual meetings of the Pediatric Academic Societies (1993 to 2017), the European Society for Paediatric Research (1995 to 2017), the Royal College of Paediatrics and Child Health (2000 to 19 February 2018), and the Perinatal Society of Australia and New Zealand (2000 to 2017). Trials reported only as abstracts were eligible if sufficient information was available from the report, or from contact with trial authors, to fulfil the inclusion criteria.

\section{Data collection and analysis}

We used the standard methods of Cochrane Neonatal and Cochrane (Higgins 2017).

\section{Selection of studies}

Two review authors (TA and ST) screened the title and abstract of all studies identified by the above search strategy and independently assessed the full-text articles for all potentially relevant trials. We excluded those studies that did not meet all of the inclusion criteria, and we stated the reasons for exclusion. We discussed any disagreements until consensus was achieved.

We recorded the selection process in sufficient detail to complete a PRISMA flow diagram and a Characteristics of excluded studies table (Moher 2009).

\section{Data extraction and management}

Two review authors (TA and ST) extracted data independently using a data collection form to aid extraction of information on design, methodology, participants, interventions, outcomes, and treatment effects from each included study. We discussed any disagreements until we reached a consensus. If data from the trial reports were insufficient, we contacted the trialists for further information.

\section{Assessment of risk of bias in included studies}

Two review authors (TA and ST) independently assessed risk of bias (low, high, or unclear) of all included trials using the Cochrane 'Risk of bias' tool for the following domains (Higgins 2017).

- Sequence generation (selection bias)

- Allocation concealment (selection bias)

- Blinding of participants and personnel (performance bias)

- Blinding of outcome assessment (detection bias)

- Incomplete outcome data (attrition bias)

- Selective reporting (reporting bias)

- Any other bias

We discussed any disagreements until we reached a consensus. See Appendix 2 for a more detailed description of risk of bias for each domain.

\section{Measures of treatment effect}

We analysed treatment effects in the individual trials using RevMan 2014 and reported risk ratio (RR) and risk difference (RD) for dichotomous data, and mean difference (MD) for continuous data, with respective $95 \%$ confidence intervals $(\mathrm{Cls})$. We determined the number needed to treat for an additional beneficial outcome (NNTB) or an additional harmful outcome (NNTH) for analyses with a statistically significant difference in the RD.

\section{Unit of analysis issues}

The unit of analysis was the participating infant in individually randomised trials. For cluster-randomised trials, we planned to undertake analysis at the level of the individual while accounting for clustering in the data using an estimate of the intracluster correlation coefficient (ICC) derived from the trial (if possible), or from another source (Higgins 2017). If ICCs from other sources were used, we planned to report this and to conduct sensitivity analyses to investigate the effect of variation in the ICC. If we identified both cluster-randomised trials and individually randomised trials, we planned to combine the results where there was little heterogeneity between study designs and few interactions between effects of the intervention, and where the choice of randomisation unit was considered unlikely.

\section{Dealing with missing data}

We requested additional data from the trialists if data on important outcomes were missing or were reported unclearly. Where data were still missing, we planned to examine the impact on effect size estimates in sensitivity analyses using the 'best-worst case scenario' technique.

\section{Assessment of heterogeneity}

We examined treatment effects of individual trials and heterogeneity between trial results by inspecting the forest plots. We calculated the $I^{2}$ statistic for each RR analysis to quantify inconsistency across studies and described the percentage of variability in effect estimates that might be due to heterogeneity rather than to sampling error. Heterogeneity was classified as none $(<25 \%)$; low $(25 \%$ to $49 \%)$; moderate $(50 \%$ to $74 \%)$; or high $(\geq 75 \%)$. If we detected moderate or high heterogeneity $\left(I^{2} \geq\right.$ $50 \%$ ), we explored the possible causes (e.g. differences in study 
design, participants, interventions, or completeness of outcome assessments).

\section{Assessment of reporting biases}

If 10 or more trials were included in a meta-analysis, we planned to examine a funnel plot for asymmetry.

\section{Data synthesis}

We analysed all infants randomised on an intention-to-treat basis and treatment effects in the individual trials using a fixed-effect model to combine the data. For meta-analyses of categorical outcomes, we calculated typical estimates of RR and RD, each with 95\% Cls; for continuous outcomes, we calculated the mean difference (MD) if outcomes were measured in the same way between trials, and standardised mean difference (SMD) to combine trials measuring the same outcome using different scales. We determined the number needed to treat for an additional beneficial outcome (NNTB) or an additional harmful outcome (NNTH) for analyses with a statistically significant difference in the RD. Where meta-analysis was judged to be inappropriate, we planned to analyse and interpret individual trials separately.

\section{Quality of evidence}

We used the GRADE approach, as outlined in the GRADE Handbook (Schünemann 2013), to assess the quality of evidence for the main comparison at the outcome level.

Two review authors (TA and ST) independently assessed the quality of the evidence for outcomes identified as critical or important for decision-making. We considered evidence from RCTs as high quality but downgraded the evidence one level for serious (or two levels for very serious) limitations based upon the following: design (risk of bias), consistency across studies, directness of the evidence, precision of estimates, and presence of publication bias. We used the GRADEpro GDT Guideline Development Tool to create a 'Summary of findings' table to report the quality of the evidence.

The GRADE approach results in an assessment of the quality of a body of evidence in one of four grades.

- High: we are very confident that the true effect lies close to that of the estimate of the effect.

- Moderate: we are moderately confident in the effect estimate: the true effect is likely to be close to the estimate of the effect, but there is a possibility that it is substantially different.
- Low: our confidence in the effect estimate is limited: the true effect may be substantially different from the estimate of the effect.

- Very low: we have very little confidence in the effect estimate: the true effect is likely to be substantially different from the estimate of effect.

\section{Subgroup analysis and investigation of heterogeneity}

- Based on gestational age: $\leq 27$ weeks', 28 weeks' to 31 weeks', $\geq$ 32 weeks'

- Based on birth weight: < 1000 g, 1000 g to 1499 g, $\geq 1500$ g

- Small for gestational age versus appropriate for gestational age infants (classified using birth weight relative to the reference population)

- Type of feed the infant is receiving (human milk vs formula)

- Frequency of monitoring of gastric residual (before every feed, before every third feed, etc.)

\section{Sensitivity analysis}

We planned to undertake sensitivity analyses to determine if the findings were affected by including only studies of adequate methodology (low risk of bias), defined as adequate randomisation and allocation concealment, blinding of intervention and measurement, and less than $10 \%$ loss to follow-up.

\section{RES U LTS}

\section{Description of studies}

See Characteristics of included studies, Characteristics of excluded studies, and Characteristics of ongoing studies.

Three studies satisfied our inclusion criteria (Kaur 2015; Torrazza 2015; Singh 2018). Two studies had analysed routine monitoring of gastric residual versus no monitoring of gastric residual and were included in comparison 1 (Kaur 2015; Torrazza 2015). Both studies were done in infants with birth weight $<1500 \mathrm{~g}$. In one study, routine monitoring of gastric residual had been done in both groups and two different criteria of gastric residual had been used for interrupting feeds (Singh 2018). Hence, the study was included in comparison 2 (Singh 2018). This study was done in infants with birth weight $\geq 1500 \mathrm{~g}$.

\section{Results of the search}

See Figure 1. 
Figure 1. Study flow diagram.

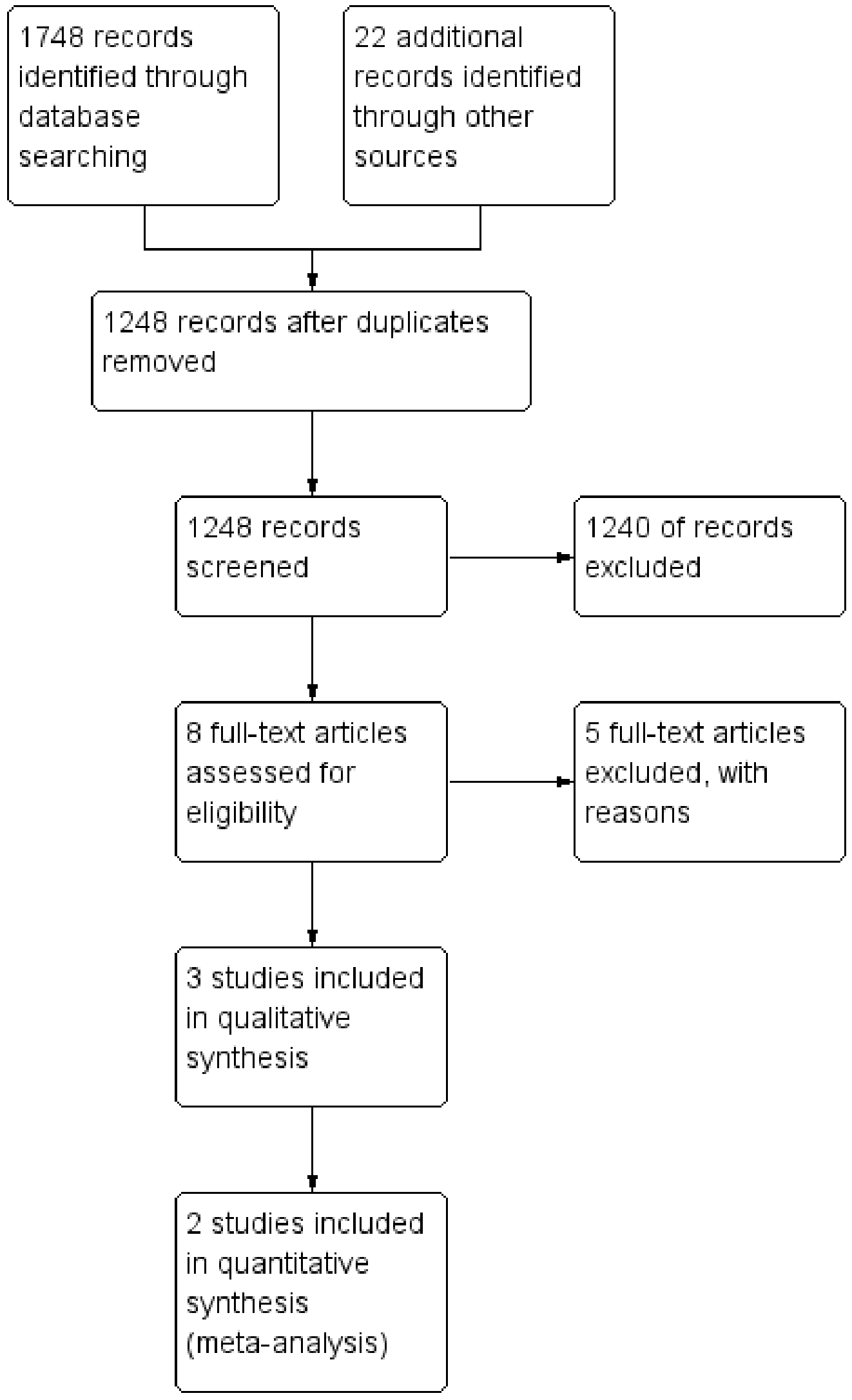




\section{Included studies}

Comparison 1. Routine monitoring of gastric residual versus no monitoring of gastric residual in preterm infants

\section{Kaur 2015}

Trialists randomised 80 infants with birth weight $<1500 \mathrm{~g}$ into the gastric residual volume monitoring group or the abdominal circumference monitoring group at the time of initiation of enteral feeds. In the gastric residual volume group, gastric residuals were measured before each feed. Feed intolerance was defined as presence of one or more of the following features: bilious/haemorrhagic aspirates or volume of aspirates $>50 \%$ of previous feed or $>3 \mathrm{~mL}$, whichever was larger. In the abdominal circumference monitoring group, abdominal girth measurements were performed before each feed. An increase in prefeed abdominal circumference by $2 \mathrm{~cm}$ from baseline was considered a sign of feed intolerance. Infants in both groups who experienced feed intolerance were kept nil per oral for the next 24 hours. Once abdominal girth was less than or equal to baseline (abdominal circumference group) or gastric aspirates were clear and $<10 \mathrm{~mL} / \mathrm{kg} / \mathrm{d}$ (gastric residual volume group), feeds were restarted at $50 \%$ of the volume being delivered at the time of feed interruption. The primary outcome was time taken to achieve full feeds $(180 \mathrm{~mL} / \mathrm{kg} / \mathrm{d})$, which were tolerated for at least 24 hours. Secondary outcomes were incidence of feed intolerance, time taken to regain birth weight, feed interruption days, duration on TPN, incidence of NEC (Bell stage 2), incidence of culture-positive sepsis, duration of hospital stay, and mortality.

\section{Torazza 2014}

Sixty-one infants at 23 to 31 weeks' gestational age with birth weight $\leq 1250 \mathrm{~g}$ who were receiving some enteral nutrition by 48 hours of age were recruited. These infants were randomised to routine monitoring of gastric residuals before every feeding or no monitoring of gastric residuals. Enteral feeds were started at $20 \mathrm{~mL} / \mathrm{kg} / \mathrm{d}$ and were increased by $20 \mathrm{~mL} / \mathrm{kg} / \mathrm{d}$. Both human milk and preterm formula were used for feeding. Abdominal distension/discolouration/tenderness, emesis, gastric residual > $50 \%$ of the feed volume or bilious aspirates were taken as signs of feed intolerance and an abdominal radiograph was taken. If the radiograph was normal, feeds were continued, and increasing the length of feeds to 30 to 50 minutes, decreasing feed volume, or changing to continuous feeds was considered. If the radiograph was abnormal, feeds were withheld for 24 hours followed by reassessment. Primary outcomes were enteral intake at two weeks and days to reach $120 \mathrm{~mL} / \mathrm{kg} / \mathrm{d}$ of enteral feedings. Secondary outcomes were enteral intake at three weeks, days to reach $150 \mathrm{~mL} /$ $\mathrm{kg} / \mathrm{d}$, growth indices (weight, head circumference, and length) at three weeks, TPN days, CVL days, and incidence of NEC, sepsis, and parental nutrition-associated liver disease.

\section{Comparison 2. Using two different criteria of gastric residual for feed interruption while monitoring gastric residual in preterm infants}

\section{Singh 2018}

Trialists recruited 87 infants with birth weight 1500 to 2000 $\mathrm{g}$ and postnatal age $<48$ hours who required gavage feeds. Routine assessment of gastric residual was done in both groups. In the intervention group, only the quality of gastric residual was assessed. A maximum of $0.5 \mathrm{~mL}$ of gastric contents was aspirated before each feed. If the residual was haemorrhagic or was repeatedly bilious (more than one time), feed interruption was done. The volume of gastric residual was not assessed. In the control group, both volume and quality of gastric residual were assessed. The entire volume of gastric residual was aspirated before every feed. If the aspirate was $>50 \%$ of feed volume or $>3$ $\mathrm{mL}$, whichever was greater, feeds were withheld. Also if the aspirate was bloody or bile stained, feeds were withheld. Other signs such as vomiting and abnormal abdominal examination were monitored in both groups. The primary outcome was time to reach full enteral feeding $\geq 120 \mathrm{~mL} / \mathrm{kg} / \mathrm{d}$. Secondary outcomes were time to regain birth weight, time to regain $120 \%$ of birth weight, incidence of lateonset culture-proven sepsis ( $\geq 72$ hours), NEC (Bell stage $\geq 2$ ), and number of occasions feedings either were discontinued or were not increased for $>24$ hours.

\section{Excluded studies}

We excluded five studies (see Characteristics of excluded studies). Three were case-control studies (Cobb 2004; Bertino 2009; Riskin 2017). Bertino 2009 and Cobb 2004 matched infants with NEC with control infants and studied the role of gastric residuals in early identification of NEC. Riskin 2017 evaluated the time to full enteral feedings and the incidence of NEC in preterm infants after a practice change from routine evaluation of gastric residual volume before each feeding to selective evaluation of gastric residual volume.

Two were observational studies (Malhotra 1992; Mihatsch 2002). Malhotra 1992 studied the volume of gastric residual in healthy preterm infants prospectively and analysed the various factors influencing gastric residual such as postnatal age, position of the baby, type of milk, and small for gestational age. Mihatsch 2002 evaluated whether the volume of gastric residual and bilious gastric residual was a significant predictor of feeding intolerance in extremely low birth weight infants.

\section{Risk of bias in included studies}

See Figure 2. 
Figure 2. Risk of bias summary: review authors' judgements about each risk of bias item for each included study.

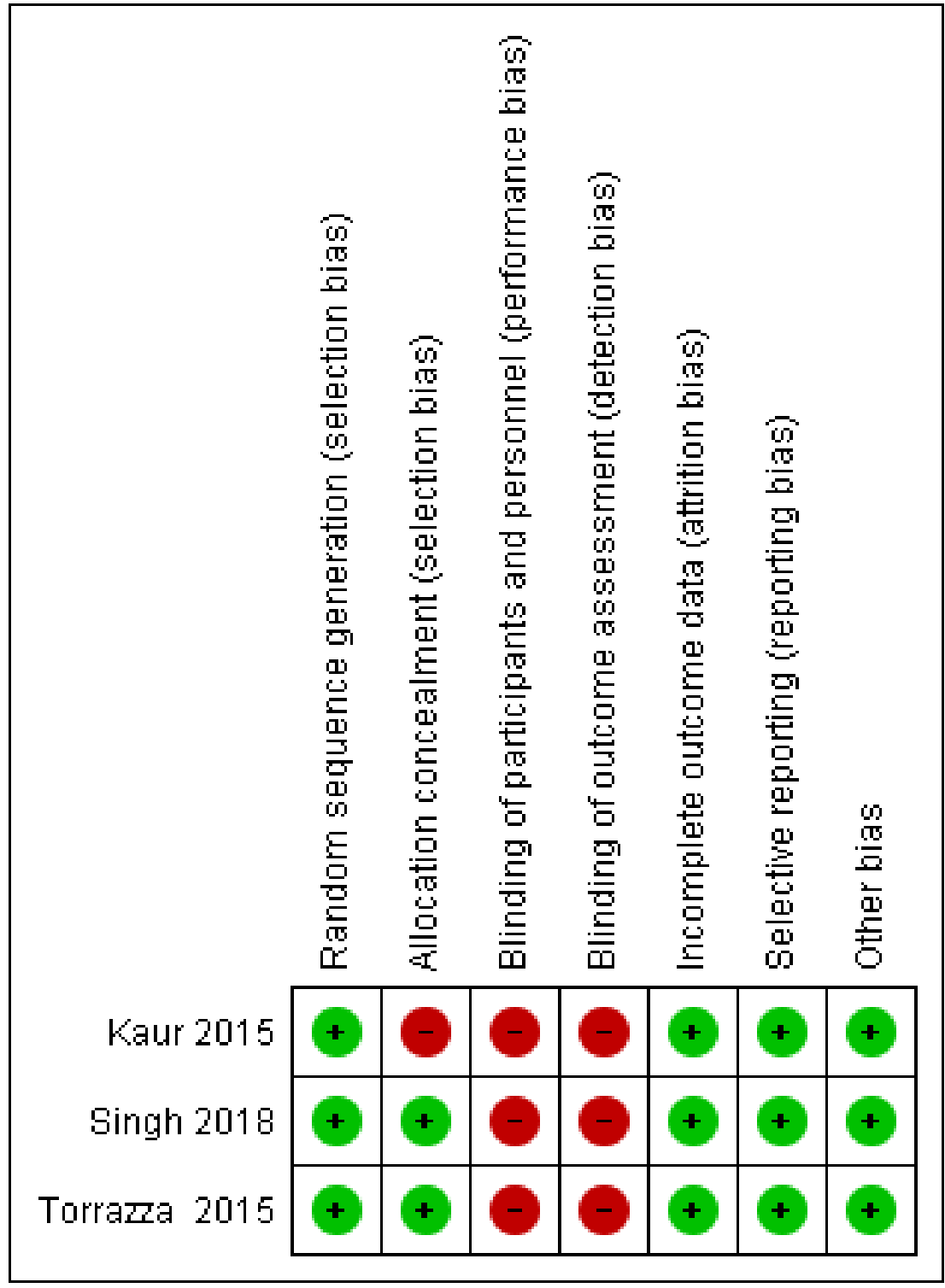

\section{Allocation}

All three included studies used computer-generated random number sequence and used serially numbered opaque envelopes to conceal the allocation (Kaur 2015; Torrazza 2015; Singh 2018). However, allocation concealment was inadequate in one study due to the fixed block size used (Kaur 2015). In this open-label trial, a fixed block size of four made the allocation of every fourth infant predictable.

\section{Blinding}

Due to the nature of the intervention, caregivers in all three trials could not be masked (Kaur 2015; Torrazza 2015; Singh 2018).

\section{Incomplete outcome data}

All recruited infants were accounted for in all three studies (Kaur 2015; Torrazza 2015; Singh 2018).

\section{Selective reporting}

All proposed outcomes were reported in all three studies (Kaur 2015; Torrazza 2015; Singh 2018).

\section{Other potential sources of bias}

No other potential source of bias was noted in any of the included studies (Kaur 2015; Torrazza 2015; Singh 2018).

\section{Effects of interventions}

See: Summary of findings for the main comparison Routine monitoring of gastric residuals compared to no routine monitoring of gastric residuals for prevention of necrotising enterocolitis in preterm infants

See Summary of findings for the main comparison. One hundred forty-one infants from two randomised trials were included in the meta-analysis to assess the effects of routine monitoring of gastric residual versus no monitoring on various outcomes in 
preterm infants. The quality of evidence was low for all outcomes, downgraded for risk of bias and/or imprecise estimates.

One trial with 87 infants was included to assess the effects of using two different criteria of gastric residual for feed interruption while monitoring gastric residual in preterm infants.
Comparison 1. Routine monitoring of gastric residual versus no monitoring of gastric residual

\section{NEC stage 2 or 3}

(Analysis 1.1; Figure 3)

Figure 3. Forest plot of comparison: 1 Routine monitoring of gastric residuals versus no monitoring of gastric residuals, outcome: 1.1 Number of infants with NEC stage 2 or 3.

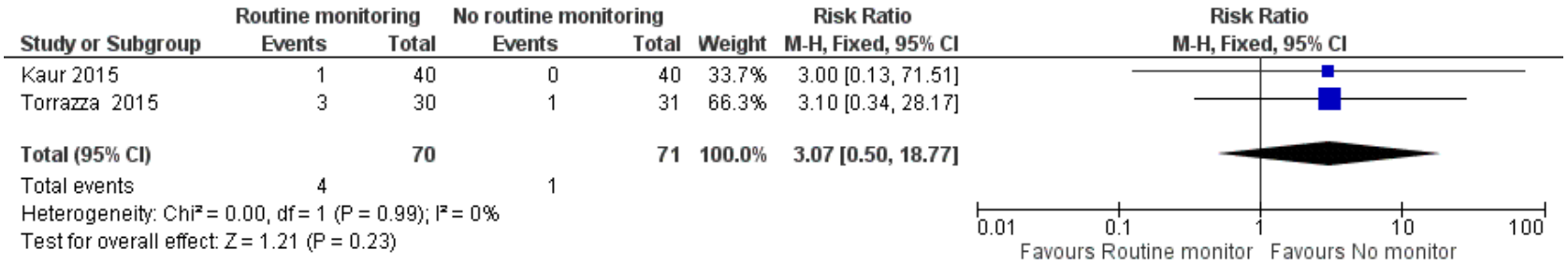

Data were available from both trials and 141 participants for analysis of this outcome (Kaur 2015; Torrazza 2015). The metaanalysis did not show a difference in the incidence of NEC between routine monitoring of gastric residual and no monitoring (RR 3.07,
$95 \% \mathrm{Cl} 0.50$ to 18.77 ; participants $=141$; studies $=2)$. There was no evidence of heterogeneity $\left(I^{2}=0 \%\right)$.

\section{Time to establish full enteral feeds}

(Analysis 1.2; Figure 4)

Figure 4. Forest plot of comparison: 1 Routine monitoring of gastric residuals versus no monitoring of gastric residuals, outcome: 1.2 Time to reach full enteral feeds.

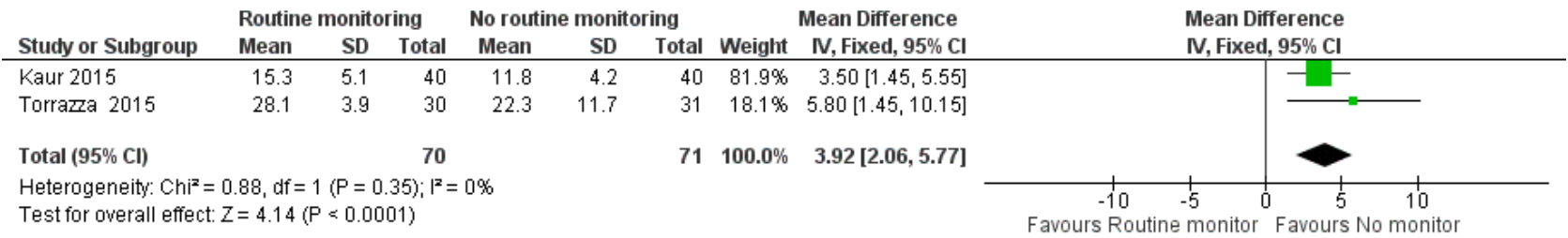

Both trials reported the outcome of time to establish full enteral feeds $\geq 150 \mathrm{~mL} / \mathrm{kg} / \mathrm{d}$ (Kaur 2015; Torrazza 2015). The meta-analysis showed that routine monitoring of gastric residual delays the time to establish full enteral feeds by 3.9 days (MD 3.92, 95\% $\mathrm{Cl} 2.06$ to 5.77 days; participants $=141$; studies $=2)$. There was no evidence of heterogeneity $\left(I^{2}=0 \%\right)$.

\section{Surgical NEC}

(Analysis 1.3)
Data from one trial were available for assessment of this outcome (Kaur 2015). The trial showed no difference in the incidence of surgical NEC between routine monitoring and no monitoring (RR $1.25,95 \% \mathrm{Cl} 0.36$ to 4.32 ; participants $=80$; studies $=1$ ).

\section{Time to regain birth weight}

(Analysis 1.4; Figure 5)

Figure 5. Forest plot of comparison: 1 Routine monitoring of gastric residuals versus no monitoring of gastric residuals, outcome: 1.4 Time to regain birth weight (days).

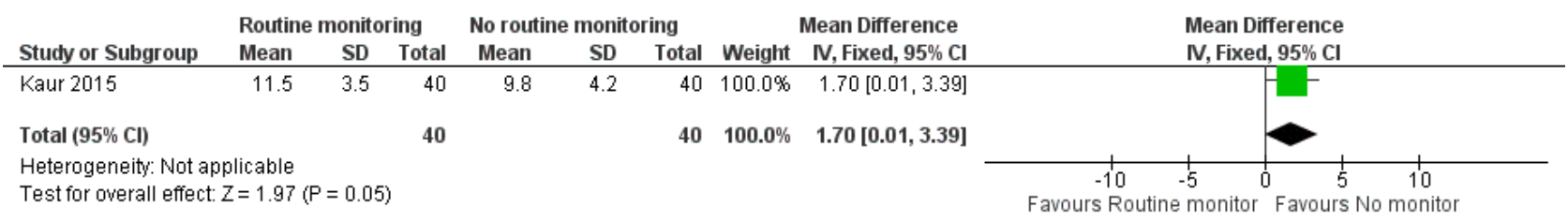

Data from one trial showed that infants in the routine monitoring group regained birth weight later by 1.7 days compared to the no monitoring group (MD 1.70, 95\% $\mathrm{Cl} 0.01$ to 3.39 days; participants = 80 ; studies $=1)($ Kaur 2015).

\section{Extrauterine growth restriction at discharge}

(Analysis 1.5)

There was no difference in the incidence of extrauterine growth restriction at discharge between groups based on data from one 
trial (RR 0.89, 95\% Cl 0.75 to 1.05; participants = 80; studies $=1$ ) (Kaur 2015).
Number of infants with episodes of feed interruption $\geq 12$ hours

(Analysis 1.6; Figure 6)

Figure 6. Forest plot of comparison: 1 Routine monitoring of gastric residuals versus no monitoring of gastric residuals, outcome: 1.6 Number of infants with episodes of interruption of feeds (lasting $\geq 12$ hours).

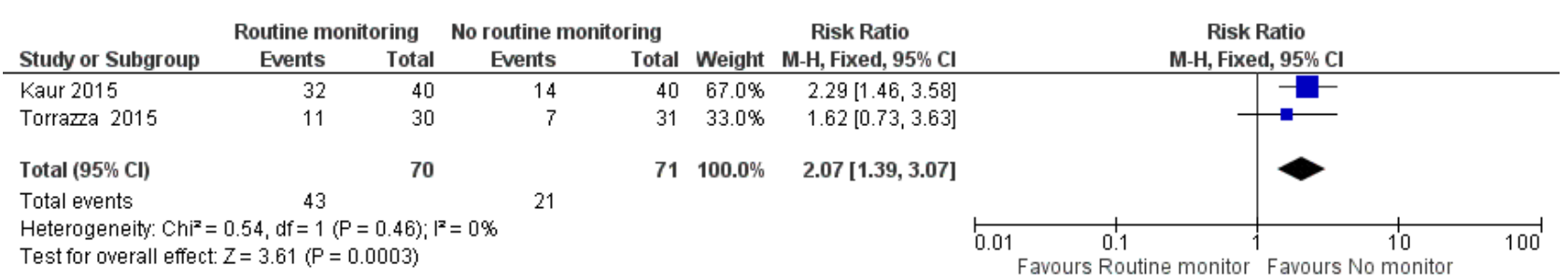

Data from both trials were available for this outcome (Kaur 2015; Torrazza 2015). There was a significant increase in the episodes of feed interruption in the routine monitoring group (RR 2.07, 95\% $\mathrm{Cl} 1.39$ to 3.07 ; participants $=141$; studies $=2$ ). Heterogeneity was moderate $\left(I^{2}=66 \%\right)$.
Number of TPN days

(Analysis 1.7; Figure 7)

Figure 7. Forest plot of comparison: 1 Routine monitoring of gastric residuals versus no monitoring of gastric residuals, outcome: 1.7 Number of TPN days.

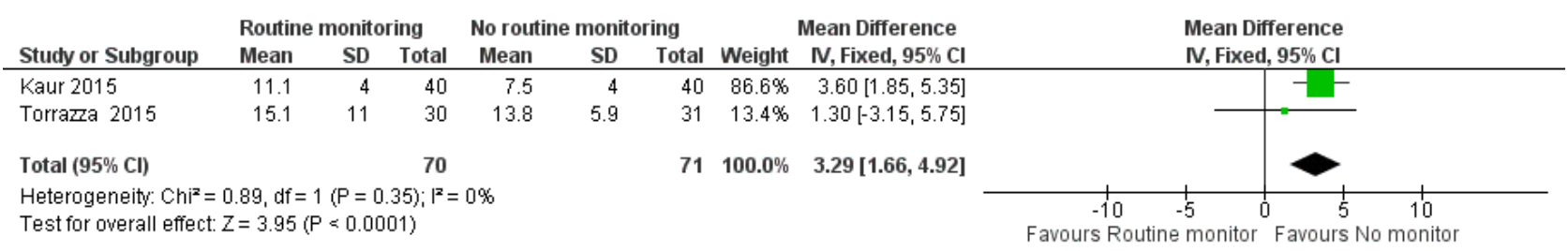

There was a significant increase in the number of TPN days in the routine monitoring group compared to the no monitoring group based on data from both trials (MD 3.29, 95\% Cl 1.66 to 4.92 days; participants $=141$; studies $=2)($ Kaur 2015; Torrazza 2015).

\section{Parenteral nutrition-associated liver disease}

\section{(Analysis 1.8)}

Data from one trial showed no difference in the incidence of parenteral nutrition-associated liver disease between groups (RR $1.03,95 \% \mathrm{Cl} 0.28$ to 3.76 ; participants $=61$; studies $=1)($ Torrazza 2015).

\section{Duration of central venous line (CVL) usage}

(Analysis 1.9)

Data were available from one trial (Torrazza 2015). There was no significant difference in the duration of CVL usage between routine monitoring of gastric residual and no monitoring (MD 5.70, 95\% Cl -1.99 to 13.39 days; participants $=61$; studies $=1$ ).

\section{Incidence of invasive infection}

(Analysis 1.10)

Data were available from both trials for analysis of this outcome (Kaur 2015; Torrazza 2015; Singh 2018). There was no significant difference in the incidence of invasive infection between routine monitoring of gastric residual and no monitoring (RR $1.46,95 \% \mathrm{Cl}$ 0.85 to 2.52 ; participants $=141$; studies $=2$ ) .

\section{Aspiration pneumonia}

Neither of the trials reported aspiration pneumonia as an outcome.

\section{Gastroesophageal reflux}

Neither of the trials reported gastroesophageal reflux as an outcome.

\section{All-cause mortality before discharge}

(Analysis 1.11)

One trial showed no significant difference in mortality before discharge between the routine monitoring and no monitoring groups (RR 3.21, $95 \% \mathrm{Cl} 0.13$ to 76.67 ; participants $=87$; studies $=1$ ) (Kaur 2015).

\section{Duration of hospital stay}

(Analysis 1.12)

Data were available from only one trial (Kaur 2015). There was no significant difference in the duration of hospital stay between the routine monitoring and no monitoring groups (MD 3.20, 95\% Cl -4.45 to 10.85 days; participants $=80$; studies $=1$ ).

\section{Growth measures following discharge}

Neither of the trials assessed growth measures following discharge. 


\section{Neurodevelopmental outcomes}

Neither of the trials assessed neurodevelopmental outcomes.

Comparison 2. Using two different criteria of gastric residual for feed interruption while monitoring gastric residual

\section{NEC stage 2 or 3}

(Analysis 2.1)

The trial did not show a difference in the incidence of NEC between groups with interruption of feeds based on quality and volume and based on quality alone (RR 5.35, 95\% Cl 0.26 to 108.27 ; participants $=87$; studies $=1)($ Singh 2018).

\section{Time to establish full enteral feeds}

(Analysis 2.2)

The trial did not show a significant difference in time to reach full enteral feeds (MD $-0.10,95 \% \mathrm{Cl}-0.91$ to 0.71 days; participants $=87$; studies $=1)($ Singh 2018).

\section{Surgical NEC}

\section{(Analysis 2.3)}

The trial showed no difference in surgical NEC (RR 5.35, 95\% Cl 0.26 to 108.27 ; participants $=87$; studies $=1)($ Singh 2018) .

\section{Time to regain birth weight}

\section{(Analysis 2.4)}

There was no difference in time to regain birth weight between groups (MD 1.00, $95 \% \mathrm{Cl}-0.37$ to 2.37 days; participants $=87$; studies = 1) (Singh 2018).

\section{Extrauterine growth restriction at discharge}

(Analysis 2.5)

There was no difference in the incidence of extrauterine growth restriction between two groups (RR $0.54,95 \% \mathrm{Cl} 0.14$ to 2.01 ; participants $=87$; studies $=1)($ Singh 2018).

\section{Number of infants with episodes of feed interruption $\geq 12$ hours}

(Analysis 2.6)

There was no difference in the number of infants with episodes of feed interruption between groups (RR $3.21,95 \% \mathrm{Cl} 0.13$ to 76.67 ; participants $=87$; studies $=1)($ Singh 2018).

\section{Number of TPN days}

\section{(Analysis 2.7)}

Trial data showed no difference in the number of TPN days between groups ( $\mathrm{MD} 0.80,95 \% \mathrm{Cl}-0.78$ to 2.38 days; participants $=87$; studies = 1) (Singh 2018).

\section{Parenteral nutrition-associated liver disease}

None of the infants in either group developed parenteral nutritionassociated liver disease (Singh 2018).

\section{Duration of central venous line (CVL) usage}

The trial did not report CVL days as an outcome (Singh 2018).

\section{Incidence of invasive infection}

(Analysis 2.8)

There was no significant difference in the incidence of invasive infection between groups (RR 5.35, 95\% Cl 0.26 to 108.27; participants $=87$; studies $=1)($ Singh 2018).

\section{Aspiration pneumonia}

The trial did not report aspiration pneumonia as an outcome (Singh 2018).

\section{Gastroesophageal reflux}

The trial did not report gastroesophageal reflux as an outcome (Singh 2018).

\section{All-cause mortality before discharge}

(Analysis 2.9)

Trial data did not show any difference in mortality before discharge $($ RR 3.21, 95\% Cl 0.13 to 76.67 ; participants = 87; studies $=1)($ Singh 2018).

\section{Duration of hospital stay}

The trial did not report duration of hospital stay as an outcome (Singh 2018).

\section{Growth measures following discharge}

The trial did not assess growth measures following discharge (Singh 2018).

\section{Neurodevelopmental outcomes}

The trial did not assess neurodevelopmental outcomes (Singh 2018).

\section{Subgroup analyses}

Based on gestational age ( $\leq 27$ weeks, 28 weeks to 31 weeks, $\geq$ 32 weeks): this subgroup analysis was not possible. One trial for comparison 1 included infants at 23 to 32 weeks' gestational age (Torrazza 2015), and the other included infants at 27 to 34 weeks' gestational age (Kaur 2015). The only trial in comparison 2 used only birth weight and did not use gestational age criteria for recruitment (Singh 2018).

Based on birth weight (<1000 g, $1000 \mathrm{~g}$ to $1499 \mathrm{~g}$, $\geq 1500 \mathrm{~g}$ ): this subgroup analysis was not possible. Both trials for comparison 1 included babies with < 1500 g birth weight (Kaur 2015; Torrazza 2015), and the only trial for comparison 2 included only infants at $\geq$ $1500 \mathrm{~g}$ birth weight (Singh 2018).

Small for gestational age versus appropriate for gestational age infants (classified using birth weight relative to the reference population): this subgroup analysis was not possible. Both trials for comparison 1 did not provide data on SGA infants separately (Kaur 2015; Torrazza 2015), and the trial for comparison 2 excluded infants with birth weight below the third percentile (Singh 2018). 
Type of feed the infant is receiving (human milk vs formula): this subgroup analysis was not possible. All included trials used human milk and/or formula to feed the infants (Kaur 2015; Torrazza 2015; Singh 2018).

Frequency of monitoring of gastric residual (before every feed, before every third feed, etc.): this subgroup analysis was not possible. All included trials monitored gastric residual before every feed (Kaur 2015; Torrazza 2015; Singh 2018).

\section{DISCUSSION}

\section{Summary of main results}

Two randomised controlled trials (RCTs) met the inclusion criteria for the comparison of routine monitoring versus no monitoring of gastric residual in preterm infants (Kaur 2015; Torrazza 2015); both trials were done in infants $<1500 \mathrm{~g}$. These trials were unblinded but otherwise of good methodological quality. However, estimates were imprecise due to the small sample size.

Routine monitoring of gastric residual may have little or no effect on the incidence of necrotising enterocolitis (risk ratio (RR) 3.07, 95\% confidence interval $(\mathrm{Cl}) 0.50$ to 18.77 ; participants $=141$; studies $=2$; low-quality evidence). Routine monitoring may increase the risk of feed interruption episodes (RR 2.07, 95\% Cl 1.39 to 3.07; participants $=141$; studies $=2$; low-quality evidence); the number needed to treat for an additional harmful outcome (NNTH) was 3 (95\% $\mathrm{Cl} 2$ to 6$)$.

Routine monitoring of gastric residual may increase time taken to establish full enteral feeds (mean difference (MD) 3.92, 95\% Cl 2.06 to 5.77 days; participants $=141$; studies $=2$; low-quality evidence), time taken to regain birth weight (MD 1.70, 95\% Cl 0.01 to 3.39 days; participants $=80$; studies $=1$; low-quality evidence), and number of total parenteral nutrition days (MD 3.29, $95 \% \mathrm{Cl} 1.66$ to 4.92 days; participants $=141$; studies $=2$; low-quality evidence).

We are uncertain as to the effect of routine monitoring of gastric residual on other outcomes such as incidence of surgical necrotising enterocolitis, extrauterine growth restriction at discharge, parenteral nutrition-associated liver disease, duration of central venous line $(\mathrm{CVL})$ usage, incidence of invasive infection, mortality before discharge, and duration of hospital stay. We found no data for outcomes such as aspiration pneumonia, gastroesophageal reflux, growth measures following discharge, and neurodevelopmental outcome. The three ongoing trials could provide more data on important outcomes of routine monitoring of gastric residual versus no monitoring in preterm infants (Parker 2013; Aljariry 2017; GRASS 2017).

One trial met the inclusion criteria for the comparison of using two different criteria of gastric residual for interrupting feeds, while gastric residual monitoring was done in both groups (Singh 2018). The trial was done in infants $\geq 1500 \mathrm{~g}$. The trial was unblinded but otherwise of good methodological quality. In this trial, both quality and volume of gastric residual were monitored in the intervention group; interruption of feeds and/or decreasing the feed volume was done considering both quality and volume of the residual. In the control group, only the quality of gastric residual was monitored and considered for feed interruption; the volume of gastric residual was not monitored.
We are uncertain as to the effect of using two different criteria of gastric residual on outcomes such as incidence of necrotising enterocolitis or surgical necrotising enterocolitis, time to establish full enteral feeds, time to regain birth weight, number of total parenteral nutrition days, number of infants experiencing feed interruption episodes, extrauterine growth restriction at discharge, parenteral nutrition-associated liver disease, incidence of invasive infection, and mortality before discharge (very low quality evidence). We found on data on duration of CVL usage, aspiration pneumonia, gastroesophageal reflux, duration of hospital stay, growth measures following discharge, and neurodevelopmental outcome.

\section{Overall completeness and applicability of evidence}

For the comparison of routine monitoring versus no monitoring of gastric residual, both included trials were done in infants with birth weight < $1500 \mathrm{~g}$ (Kaur 2015; Torrazza 2015). One trial excluded infants with absent or reversed end-diastolic flow in antenatal doppler and infants with perinatal asphyxia (Kaur 2015). Study infants were given intermittent gavage feeds with human milk or formula milk in both trials. Trials had predefined criteria to interrupt feeds, although these criteria varied slightly between trials.

In one trial, there was no proactive monitoring for feed intolerance in the 'no monitoring' group (Torrazza 2015). Kaur 2015 monitored the prefeed abdominal circumference proactively and checked gastric residual whenever there was an increase in abdominal circumference $>2 \mathrm{~cm}$. Hence the comparison group was not similar in the two included trials.

The meta-analysis shows that routine monitoring of gastric residual may increase the time taken to reach full enteral feeds $\geq 150 \mathrm{~mL} /$ $\mathrm{kg} / \mathrm{d}$, the time taken to regain birth weight, and the number of TPN days, and may increase the number of infants experiencing feed interruption episodes. Although not shown in this meta-analysis, a decrease in the number of TPN days would decrease the number of days of CVL usage, risk of invasive infection, parenteral nutritionassociated liver disease, and duration of hospital stay.

The major concern in not monitoring gastric residual is that it takes away an early indicator of NEC and hence may increase the risk of NEC. However, this meta-analysis shows a trend towards increased risk of NEC in the routine monitoring group. This could be due to over-diagnosis of NEC in the routine monitoring group based on bloody aspirates caused by mucosal trauma due to repeated aspirations or based on benign bilious aspirates in preterm infants. In any case, there is no evidence to say that not monitoring gastric residual in preterm infants would increase the risk of NEC.

For the comparison of using two different criteria of gastric residual to interrupt feeds while monitoring gastric residual, the only included trial was done in preterm infants with birth weight of 1500 to $2000 \mathrm{~g}$. The trial excluded infants with perinatal asphyxia and birth weight below the third percentile. The trial showed no difference in any of the major outcomes such as NEC, time to reach full enteral feeds, time taken to regain birth weight, number of episodes of feed interruption, and number of TPN days. This could be so because these larger preterm infants usually do not require long duration of gavage feeds, TPN, or CVL usage, and they are at lesser risk of NEC when compared to very low birth weight infants. 


\section{Quality of the evidence}

The methodological quality of the included trials was good; trialists used randomisation and allocation concealment to avoid selection bias, reported all intended outcomes, and there was no attrition. However, none of the trials was blinded.

The quality of evidence was low for all outcomes: NEC (downgraded for serious imprecision due to small sample size and low event rate), time to establish full enteral feeds, time to regain birth weight, and number of TPN days (downgraded for serious imprecision due to small sample size), and number of feed interruption episodes (downgraded for imprecision due to small sample size and lack of blinding). Clinicians' assessment of feed intolerance and decision to withhold feeds are subjective; hence there is always a risk of surveillance and ascertainment bias in an unblinded trial.

For the comparison of two different criteria of gastric residual to interrupt feeds, the quality of evidence from the only included trial was very low for all outcomes (downgraded for serious imprecision due to the small sample size and wide confidence intervals and indirectness because the trial was done in infants with 1500 to 2000 g birth weight).

\section{Potential biases in the review process}

We have no financial or other conflicts of interest.

We found only three small trials for inclusion in this review. Although we conducted a comprehensive search, we cannot exclude fully the possibility of publication bias because we do not know whether other published (but not indexed) or unpublished trials have been conducted. We did not have a sufficient number of trials to explore symmetry of funnel plots as a means of identifying possible publication or reporting bias.

\section{Agreements and disagreements with other studies or reviews}

To the best of our knowledge, there is no other systematic review on routine monitoring of gastric residual for prevention of NEC in preterm infants. Riskin 2017 is a case-control study done in 472 preterm infants < 34 weeks' gestation on the effects of routine monitoring of gastric residual versus no monitoring. The study showed no difference in the incidence of NEC with routine monitoring of gastric residual. Routine monitoring increased the risk of feed interruption episodes, increased the time to reach full enteral feeds and number of TPN days, reduced weight gain and weight at discharge and increased the postmenstrual age at discharge. Thus, the results of the study were similar to our metaanalysis.

\section{AUTHORS' CONCLUSIONS}

\section{Implications for practice}

Review authors found insufficient evidence as to whether routine monitoring of gastric residual reduces the incidence of necrotising enterocolitis because trial results are imprecise. Low-quality evidence suggests that routine monitoring of gastric residual increases the risk of feed interruption episodes, increases the time taken to reach full enteral feeds and to regain birth weight, and increases the number of total parenteral nutrition days. Available data are insufficient to comment on other major outcomes such as incidence of invasive infection, parenteral nutrition-associated liver disease, mortality before discharge, extrauterine growth restriction at discharge, number of central venous line, days and duration of hospital stay.

For the comparison of using two different criteria of gastric residual to interrupt feeds, available data are insufficient to comment on any of the major outcomes of preterm infants.

\section{Implications for research}

Further randomised controlled trials on routine monitoring of gastric residuals versus no monitoring should be adequately powered to detect significant differences in major outcomes such as necrotising enterocolitis (NEC) and mortality before discharge. These trials should provide more precise estimates on important outcomes such as incidence of invasive infection, extrauterine growth restriction at discharge, parenteral nutrition-associated liver disease, number of central venous line (CVL) days, and duration of hospital stay.

The trials should also provide data on long-term growth and neurodevelopmental outcomes. Trialists should aim to include infants with intrauterine growth restriction and perinatal asphyxia, so that subgroup analyses can be planned for this population at higher risk of NEC.

Further randomised controlled trials comparing two different criteria of gastric residual to interrupt feeds (based on quality and/or quantity) are required to provide adequate data on major outcomes in preterm infants. No uniform standards on the quality or quantity of gastric residual can predict NEC; therefore, the trialists could choose criteria from available evidence based on observational studies. Trials should provide clear evidence on the nature of gastric residual (based on quality and/or quantity) that predicts NEC.

\section{ACK N OWLEDGEMENTS}

The methods section of this protocol is based on a standard template used by Cochrane Neonatal.

We are grateful to Drs. Satish Saluja, Josef Neu, Leslie Ann Parker, Roberto Murgas Torrazza, Balpreet Singh, and Sumesh Thomas for providing further details and data. 
R E F E R E N C E S

\section{References to studies included in this review}

Kaur 2015 \{published and unpublished data\}

Kaur A, Kler N, Saluja S, Modi M, Soni A, Thakur A, et al. Abdominal circumference or gastric residual volume as measure of feed intolerance in VLBW infants. Journal of Pediatric Gastroenterology and Nutrition 2015;60(2):259-63. [DOI: 10.1097/MPG.0000000000000576; PUBMED: 25238118]

Singh 2018 \{published and unpublished data\}

Singh B, Rochow N, Chessell L, Wilson J, Cunningham K, Fusch $C$, et al. Gastric residual volume in feeding advancement in preterm infants (GRIP Study): a randomized trial. Journal of Pediatrics 2018;200:79-83. [DOI: 10.1016/j.jpeds.2018.04.072; PUBMED: 29866595]

\section{Torrazza 2015 \{published and unpublished data\}}

Torrazza RM, Parker LA, Li Y, Talaga E, Shuster J, Neu J. The value of routine evaluation of gastric residuals in very low birth weight infants. Journal of Perinatology 2015;35(1):57-60. [DOI: 10.1038/jp.2014.147; PUBMED: 25166623]

\section{References to studies excluded from this review}

Bertino 2009 \{published data only\}

Bertino E, Giuliani F, Prandi G, Coscia A, Martano C, Fabris C. Necrotizing enterocolitis: risk factor analysis and role of gastric residuals in very low birth weight infants. Journal of Pediatric Gastroenterology and Nutrition 2009;48(4):437-42. [PUBMED: 19330932]

\section{Cobb 2004 \{published data only\}}

Cobb BA, Carlo WA, Ambalavanan N. Gastric residuals and their relationship to necrotizing enterocolitis in very low birth weight infants. Pediatrics 2004;113(1 (Pt 1)):50-3. [PUBMED: 14702446]

\section{Malhotra 1992 \{published data only\}}

Malhotra AK, Deorari AK, Paul VK, Bagga A, Singh M. Gastric residuals in preterm babies. Journal of Tropical Pediatrics 1992;38(5):262-4. [DOI: 10.1093/tropej/38.5.262; PUBMED: $1433454]$

\section{Mihatsch 2002 \{published data only\}}

Mihatsch WA, von Schoenaich P, Fahnenstich H, Dehne N, Ebbecke $\mathrm{H}$, Plath $\mathrm{C}$, et al. The significance of gastric residuals in the early enteral feeding advancement of extremely low birth weight infants. Pediatrics 2002;109(3):457-9. [PUBMED: 11875141]

\section{Riskin 2017 \{published data only\}}

Riskin A, Cohen K, Kugelman A, Toropine A, Said W, Bader D. The impact of routine evaluation of gastric residual volumes on the time to achieve full enteral feeding in preterm infants. Journal of Pediatrics 2017;189:128-34. [DOI: 10.1016/ j.jpeds.2017.05.054; PUBMED: 28625498]

\section{References to ongoing studies}

Aljariry 2017 \{published data only\}

Routine gastric residual aspiration in preterm infants and the effect on reaching full feed. Ongoing study December 2015.

GRASS 2017 \{published data only\}

Does routine assessment of gastric residuals in preterm neonates influence time taken to reach full enteral feeding? (GRASS). Ongoing study October 2017.

Parker 2013 \{published data only\} Aspiration of residual gastric contents. Ongoing study May 2013.

\section{Additional references}

\section{Abiramalatha 2018}

Abiramalatha T, Thanigainathan S, Balakrishnan U. Re-feeding versus discarding gastric residuals to improve growth in preterm infants. Cochrane Database of Systematic Reviews 2018, Issue 1. [DOI: 10.1002/14651858.CD012940]

\section{Bartlett 2015}

Bartlett Ellis RJ, Fuehne J. Examination of accuracy in the assessment of gastric residual volume: a simulated, controlled study. JPEN. Journal of Parenteral and Enteral Nutrition 2015;39(4):434-40. [DOI: 10.1177/0148607114524230; PUBMED: 24562002]

\section{Cohen 2004}

Cohen S, Mandel D, Mimouni FB, Solovkin L, Dollberg S. Gastric residual in growing preterm infants: effect of body position. American Journal of Perinatology 2004;21(3):163-6. [DOI: 10.1055/s-2004-823778; PUBMED: 15085500]

\section{Duro 2011}

Duro D, Mitchell PD, Kalish LA, Martin C, McCarthy M, Jaksic T, et al. Risk factors for parenteral nutrition-associated liver disease following surgical therapy for necrotizing enterocolitis: a Glaser Pediatric Research Network Study. Journal of Pediatric Gastroenterology and Nutrition 2011;52(5):595-600 [Erratum in:Journal of Pediatric Gastroenterology and Nutrition 2011;53(5):583]. [DOI: 10.1097/MPG.0b013e31820e8396; PUBMED: 21464752

\section{Dutta 2015}

Dutta S, Singh B, Chessell L, Wilson J, Janes M, McDonald K, et al. Guidelines for feeding very low birth weight infants. Nutrients 2015;7(1):423-42. [DOI: 10.3390/nu7010423; PUBMED: 25580815

\section{Embleton 2013}

Embleton ND. Early nutrition and later outcomes in preterm infants. World Review of Nutrition and Dietetics 2013;106:26-32. [DOI: 10.1159/000342553; PUBMED: 23428677]

\section{Franz 2009}

Franz AR, Pohlandt F, Bode H, Mihatsch WA, Sander S, Kron M, et al. Intrauterine, early neonatal, and postdischarge growth and 
neurodevelopmental outcome at 5.4 years in extremely preterm infants after intensive neonatal nutritional support. Pediatrics 2009;123(1):e101-9. [DOI: 10.1542/peds.2008-1352; PUBMED: 19117831]

\section{Gephart 2017}

Gephart SM, Fleiner M, Kijewski A. The conNECtion between abdominal signs and necrotizing enterocolitis in infants 501 to 1500 g. Advances in Neonatal Care 2017;17(1):53-64. [DOI: 10.1097/ANC.0000000000000345; PUBMED: 27754992]

\section{GRADEpro GDT [Computer program]}

McMaster University (developed by Evidence Prime). GRADEpro GDT. Version accessed 11 September 2017. Hamilton (ON): McMaster University (developed by Evidence Prime), 2014.

\section{Gregory 2012}

Gregory KE, Connolly TC. Enteral feeding practices in the NICU: results from a 2009 Neonatal Enteral Feeding Survey. Advances in Neonatal Care 2012;12(1):46-55. [DOI: 10.1097/ ANC.0b013e3182425aab; PUBMED: 22301544]

\section{Grino 2016}

Grino CU, Miller M, Carrasquilla-Lopez V, Rojas M, Bhutanda A, Rastogi S, et al. Early prediction of severity of necrotizing enterocolitis in preterm infants: a composite scoring tool. Clinical Nursing Studies 2016;4(3):47-53. [DOI: 10.5430/ cns.v4n3p47]

\section{Hermansen 2005}

Hermansen MC, Hermansen MG. Intravascular catheter complications in the neonatal intensive care unit. Clinics in Perinatology 2005;32(1):141-56. [DOI: 10.1016/j.clp.2004.11.005; PUBMED: 15777826]

\section{Higgins 2017}

Higgins JP, Green S editor(s). Cochrane Handbook for Systematic Reviews of Interventions Version 5.2.0 (updated June 2017). The Cochrane Collaboration, 2017. Available from training.cochrane.org/handbook.

\section{Ittmann 1992}

Ittmann PI, Amarnath R, Berseth CL. Maturation of antroduodenal motor activity in preterm and term infants. Digestive Diseases and Sciences 1992;37(1):14-9. [PUBMED: 1728520]

\section{Juvé-Udina 2009}

Juvé-Udina ME, Valls-Miró C, Carreño-Granero A, MartinezEstalella G, Monterde-Prat D, Domingo-Felici CM, et al. To return or to discard? Randomised trial on gastric residual volume management. Intensive \& Critical Care Nursing 2009;25(5):258-67. [DOI: 10.1016/j.iccn.2009.06.004; PUBMED: 19615907]

\section{Kenton 2004}

Kenton AB, Fernandes CJ, Berseth CL. Gastric residuals in prediction of necrotizing enterocolitis in very low birth weight infants. Pediatrics 2004;113(6):1848-9. [PUBMED: 15173526]

\section{Leppänen 2014}

Leppänen M, Lapinleimu H, Lind A, Matomäki J, Lehtonen L, Haataja L, et al. Antenatal and postnatal growth and 5year cognitive outcome in very preterm infants. Pediatrics 2014;133(1):63-70. [DOI: 10.1542/peds.2013-1187; PUBMED: 24344103]

\section{Li 2014}

Li YF, Lin HC, Torrazza RM, Parker L, Talaga E, Neu J. Gastric residual evaluation in preterm neonates: a useful monitoring technique or a hindrance?. Pediatrics and Neonatology 2014;55(5):335-40. [DOI: 10.1016/j.pedneo.2014.02.008; PUBMED: 25129325]

\section{Lucchini 2011}

Lucchini R, Bizzarri B, Giampietro S, De Curtis M. Feeding intolerance in preterm infants. How to understand the warning signs. Journal of Maternal-fetal \& Neonatal Medicine 2011;24 Suppl 1:72-4. [DOI: 10.3109/14767058.2011.607663; PUBMED: 21892877]

\section{Moher 2009}

Moher D, Liberati A, Tetzlaff J, Altman DG, PRISMA Group. Preferred reporting items for systematic reviews and meta-analyses: the PRISMA statement. Journal of Clinical Epidemiology 2009;62(10):1006-12. [DOI: 10.1016/ j.jclinepi.2009.06.005; PUBMED: 19631508]

\section{Moore 2011}

Moore TA, Wilson ME. Feeding intolerance: a concept analysis. Advances in Neonatal Care 2011;11(3):149-54. [DOI: 10.1097/ ANC.0b013e31821ba28e; PUBMED: 21730906]

\section{Morris 1999}

Morris BH, Miller-Loncar CL, Landry SH, Smith KE, Swank PR, Denson SE. Feeding, medical factors, and developmental outcome in premature infants. Clinical Pediatrics 1999;38(8):451-7. [DOI: 10.1177/000992289903800802; PUBMED: 10456239]

\section{Parker 2015}

Parker L, Torrazza RM, Li Y, Talaga E, Shuster J, Neu J. Aspiration and evaluation of gastric residuals in the neonatal intensive care unit: state of the science. Journal of Perinatal \& Neonatal Nursing 2015;29(1):51-9. [DOI: 10.1097/JPN.0000000000000080; PUBMED: 25633400]

\section{RevMan 2014 [Computer program]}

Nordic Cochrane Centre, The Cochrane Collaboration. Review Manager 5 (RevMan 5). Version 5.3. Copenhagen: Nordic Cochrane Centre, The Cochrane Collaboration, 2014.

\section{Riezzo 2000}

Riezzo G, Indrio F, Montagna O, Tripaldi C, Laforgia N, Chiloiro M, et al. Gastric electrical activity and gastric emptying in term and preterm newborns. Neurogastroenterology and Motility 2000;12(3):223-9. [PUBMED: 10867619]

\section{Schünemann 2013}

Schünemann H, Brożek J, Guyatt G, Oxman A, editor(s). Handbook for grading the quality of evidence and the strength 
of recommendations using the GRADE approach (updated October 2013). GRADE Working Group, 2013. Available from https://gdt.gradepro.org/app/handbook/handbook.html..

\section{Shulman 2011}

Shulman RJ, Ou CN, Smith EO. Evaluation of potential factors predicting attainment of full gavage feedings in preterm infants. Neonatology 2011;99(1):38-44. [DOI: 10.1159/000302020; PUBMED: 20588069]

\section{Stevens 2016}

Stevens TP, Shields E, Campbell D, Combs A, Horgan M, La Gamma EF, et al. Variation in enteral feeding practices and growth outcomes among very premature infants: a report

\section{CHARACTERISTICS OF STUDIES}

Characteristics of included studies [ordered by study ID] from the New York State Perinatal Quality Collaborative. American Journal of Perinatology 2016;33(1):9-19. [DOI: 10.1055/ s-0035-1554794; PUBMED: 26084749]

\section{Walsh 1986}

Walsh MC, Kliegman RM. Necrotizing enterocolitis: treatment based on staging criteria. Pediatric Clinics of North America 1986;33(1):179-201. [PUBMED: 3081865]

\section{Williams 2010}

Williams TA, Leslie GD. Should gastric aspirate be discarded or retained when gastric residual volume is removed from gastric tubes?. Australian Critical Care 2010;23(4):215-7. [DOI: 10.1016/ j.aucc.2010.05.001; PUBMED: 20558081]

Kaur 2015

\begin{tabular}{ll}
\hline Methods & RCT \\
\hline Participants & $\begin{array}{l}\text { Eighty infants with birth weight }<1500 \text { g were included. Infants with major congenital abnormalities, } \\
\text { gestation }<27 \text { or }>34 \text { weeks, absent or reversed end-diastolic flow in antenatal doppler, or Apgar score } \\
<3 \text { at } 5 \text { minutes were excluded }\end{array}$
\end{tabular}

Interventions Infants were randomised into gastric residual volume monitoring group or abdominal circumference monitoring group at the time of initiation of enteral feeds

Gastric residual volume monitoring group:

Gastric residual volume was measured before each feed. Feed intolerance was defined as presence of 1 or more of the following features: bilious/haemorrhagic aspirates or volume of aspirates $>50 \%$ of previous feed or $>3 \mathrm{~mL}$, whichever was larger. If gastric residues were between $30 \%$ and $50 \%$ of previous feeds, the same volume was continued without making a daily increment. Feeds were advanced as per protocol if gastric residues were $<30 \%$ of previous feeds. The gastric residues aspirated were discarded

Abdominal circumference monitoring group:

Abdominal circumference measurement was performed before each feed using a standard, disposable non-stretchable paper tape with minimum markings of $1 \mathrm{~mm}$. The tape was positioned $1 \mathrm{~cm}$ above the umbilicus and was read along its bottom edge. A mark was made along the lower edge as reference for subsequent measurements. An increase in prefeed abdominal circumference by $2 \mathrm{~cm}$ from baseline was considered a sign of feed intolerance. Gastric residual volume assessment was not routinely performed unless the abdominal circumference increased by $>2 \mathrm{~cm}$. The decision for feed interruption was merely based on an increase in abdominal girth. The least abdominal circumference during the previous 24 hours was used as the baseline reference

Infants in both groups who experienced feed intolerance were kept nil per oral for the next 24 hours. Once abdominal circumference was less than or equal to baseline (abdominal circumference group) or gastric aspirates were clear and $<10 \mathrm{~mL} / \mathrm{kg} / \mathrm{d}$ (gastric residual volume group), feeds were restarted at $50 \%$ of the volume being delivered at the time of feed interruption

Outcomes Primary outcome:

Time taken to achieve full feeds $(180 \mathrm{~mL} / \mathrm{kg} / \mathrm{d})$, which were tolerated for at least 24 hours

Secondary outcomes: 
Kaur 2015 (Continued)

Incidence of feed intolerance, time taken to regain birth weight, feed interruption days, duration on TPN, incidence of NEC (Bell stage 2), incidence of culture-positive sepsis, duration of hospital stay, and mortality

Notes

The enrolled infants were assessed daily from birth for feed initiation. Feeds were initiated when infants were haemodynamically stable with soft abdomen and audible bowel sounds. Intermittent gavage feeds were given at 2-hourly intervals. Feed was started at $10 \mathrm{~mL} / \mathrm{kg}$ in infants $<1250 \mathrm{~g}$ and 20 $\mathrm{mL} / \mathrm{kg}$ in infants $\geq 1250 \mathrm{~g}$. Subsequent advancements were made by $20 \mathrm{~mL} / \mathrm{kg} / \mathrm{d}$ as tolerated, to a maximum volume of $180 \mathrm{~mL} / \mathrm{kg} / \mathrm{d}$. Expressed mother's milk was preferred; if not available, standard preterm formula with a calorie content of $80 \mathrm{kcal} / 100 \mathrm{~mL}$ was used. Human milk fortifier was added once infants tolerated $100 \mathrm{~mL} / \mathrm{kg} / \mathrm{d}$ feed volume

\section{Risk of bias}

\begin{tabular}{lll}
\hline Bias & Authors' judgement & Support for judgement \\
\hline $\begin{array}{l}\text { Random sequence genera- } \\
\text { tion (selection bias) }\end{array}$ & Low risk & $\begin{array}{l}\text { A computer-generated block randomisation sequence with block size of } 4 \text { was } \\
\text { prepared by a person not involved in clinical care, measurement of outcomes, } \\
\text { or analysis of data }\end{array}$
\end{tabular}

Allocation concealment $\quad$ High risk
(selection bias)

This randomisation sequence was kept in sequentially numbered sealed opaque envelopes. However, a fixed block size of 4 gives the chance to guess the allocation of every fourth infant in an unblinded study

\begin{tabular}{lll}
\hline $\begin{array}{l}\text { Blinding of participants } \\
\text { and personnel (perfor- }\end{array}$ & High risk & Unblinded \\
mance bias) & & \\
All outcomes & \\
\hline $\begin{array}{l}\text { Blinding of outcome as- } \\
\text { sessment (detection bias) }\end{array}$ & High risk & Unblinded \\
All outcomes & & \\
\end{tabular}

\begin{tabular}{lll}
\hline $\begin{array}{l}\text { Incomplete outcome data } \\
\text { (attrition bias) } \\
\text { All outcomes }\end{array}$ & Low risk & All 80 randomised infants were included in the analysis \\
\hline $\begin{array}{l}\text { Selective reporting (re- } \\
\text { porting bias) }\end{array}$ & Low risk & All proposed outcomes were reported \\
\hline Other bias & Low risk & Nil \\
\hline
\end{tabular}

Singh 2018

\begin{tabular}{ll}
\hline Methods & RCT \\
\hline Participants & $\begin{array}{l}\text { Infants with birth weight } 1500 \text { to } 2000 \mathrm{~g} \text { and postnatal age }<48 \text { hours who required gavage feeds were } \\
\text { included. Exclusion criteria were perinatal asphyxia (cord blood gas or first blood gas after birth with } \\
\mathrm{pH}<7.0 \text { or base excess }>-16 \mathrm{mmol} / \mathrm{L} \text { and Apgar score }<5 \text { at } 10 \text { minutes), major congenital malforma- } \\
\text { tions/surgical conditions that could interfere with feeding, and severe growth restriction (defined as } \\
\text { birth weight below the third percentile) }\end{array}$ \\
\hline
\end{tabular}

Interventions

Routine assessment of gastric residual was done in both groups

Intervention group: 
Only the quality of gastric residual was assessed. A maximum of $0.5 \mathrm{~mL}$ of gastric contents was aspirated before each feed. If the residual was haemorrhagic or was repeatedly bilious (more than 1 time) with or without vomiting or abnormal abdominal examination, feed interruption was done. The volume of gastric residual was not assessed

Control group:

Both volume and quality of gastric residual were assessed. The entire volume of gastric residual was aspirated before every feed. If the aspirate was $>50 \%$ of feed volume or $>3 \mathrm{~mL}$, whichever was greater, feeds were withheld. Also if the aspirate was bloody or bile stained, feeds were withheld

Outcomes
Primary outcome:
Time to reach full enteral feeding $\geq 120 \mathrm{~mL} / \mathrm{kg} / \mathrm{d}$
Secondary outcomes:
Time to regain birth weight, time to regain $120 \%$ of birth weight, incidence of late-onset culture-proven
sepsis $\geq 72$ hours), NEC (Bell stage $\geq 2$ ), number of occasions feedings were discontinued for $>24$ hours
or were not increased for $>24$ hours

\section{Risk of bias}

\begin{tabular}{|c|c|c|}
\hline Bias & Authors' judgement & Support for judgement \\
\hline $\begin{array}{l}\text { Random sequence genera- } \\
\text { tion (selection bias) }\end{array}$ & Low risk & $\begin{array}{l}\text { The randomisation sequence was computer generated and permuted, even } \\
\text { numbered; randomly varying block sizes were generated with a } 1: 1 \text { allocation } \\
\text { ratio }\end{array}$ \\
\hline $\begin{array}{l}\text { Allocation concealment } \\
\text { (selection bias) }\end{array}$ & Low risk & $\begin{array}{l}\text { The allocation sequence was concealed using serially numbered opaque } \\
\text { sealed envelopes }\end{array}$ \\
\hline $\begin{array}{l}\text { Blinding of participants } \\
\text { and personnel (perfor- } \\
\text { mance bias) } \\
\text { All outcomes }\end{array}$ & High risk & Unmasked trial \\
\hline $\begin{array}{l}\text { Blinding of outcome as- } \\
\text { sessment (detection bias) } \\
\text { All outcomes }\end{array}$ & High risk & Unmasked trial \\
\hline $\begin{array}{l}\text { Incomplete outcome data } \\
\text { (attrition bias) } \\
\text { All outcomes }\end{array}$ & Low risk & All 87 randomised infants were included in the analysis \\
\hline $\begin{array}{l}\text { Selective reporting (re- } \\
\text { porting bias) }\end{array}$ & Low risk & Study protocol had been published. All proposed outcomes were reported \\
\hline Other bias & Low risk & Nil \\
\hline
\end{tabular}


Torrazza 2015

\begin{tabular}{|c|c|}
\hline Methods & $\mathrm{RCT}$ \\
\hline Participants & $\begin{array}{l}\text { Sixty-one infants born at postmenstrual age }>23 \text { weeks but } \leq 32 \text { weeks with birth weight } \leq 1250 \mathrm{~g} \text { and } \\
\text { without congenital or chromosomal anomalies or gastrointestinal malformations who were receiving } \\
\text { some enteral nutrition by } 48 \text { hours of age }\end{array}$ \\
\hline \multirow[t]{3}{*}{ Interventions } & Infants were randomised before 48 hours of life to: \\
\hline & - Routine monitoring of gastric residuals before every feeding \\
\hline & - No monitoring of gastric residuals \\
\hline \multirow[t]{4}{*}{ Outcomes } & Primary outcome: \\
\hline & Enteral intake at 2 weeks and days to reach $120 \mathrm{~mL} / \mathrm{kg} / \mathrm{d}$ of enteral feedings \\
\hline & Secondary outcomes: \\
\hline & $\begin{array}{l}\text { Enteral intake at } 3 \text { weeks, days to reach } 150 \mathrm{~mL} / \mathrm{kg} / \mathrm{d} \text {, growth indices (weight, head circumference, and } \\
\text { length) at } 3 \text { weeks, TPN days, CVL days, incidence of NEC, sepsis, and parental nutrition-associated liv- } \\
\text { er disease }\end{array}$ \\
\hline Notes & $\begin{array}{l}\text { Enteral feeds were started at } 20 \mathrm{~mL} / \mathrm{kg} / \mathrm{d} \text { and were increased by } 20 \mathrm{~mL} / \mathrm{kg} / \mathrm{d} \text {. Both human milk and } \\
\text { preterm formula were used for feeding. Abdominal distension/discolouration/tenderness, emesis, gas- } \\
\text { tric residual > } 50 \% \text { of the feed volume or bilious aspirates were taken as signs of feed intolerance, and } \\
\text { an abdominal radiograph was taken. If the radiograph was normal, feeds were continued; increasing } \\
\text { length of feeds to } 30 \text { to } 50 \text { minutes; decreasing feed volume, or changing to continuous feeds was con- } \\
\text { sidered. If the radiograph was abnormal, feeds were withheld for } 24 \text { hours followed by reassessment }\end{array}$ \\
\hline
\end{tabular}

\section{Risk of bias}

\begin{tabular}{|c|c|c|}
\hline Bias & Authors' judgement & Support for judgement \\
\hline $\begin{array}{l}\text { Random sequence genera- } \\
\text { tion (selection bias) }\end{array}$ & Low risk & $\begin{array}{l}\text { A computer-generated block randomisation sequence with variable block } \\
\text { sizes was used }\end{array}$ \\
\hline $\begin{array}{l}\text { Allocation concealment } \\
\text { (selection bias) }\end{array}$ & Low risk & $\begin{array}{l}\text { The randomisation sequence was kept in sequentially numbered sealed } \\
\text { opaque envelopes }\end{array}$ \\
\hline $\begin{array}{l}\text { Blinding of participants } \\
\text { and personnel (perfor- } \\
\text { mance bias) } \\
\text { All outcomes }\end{array}$ & High risk & Open-label trial \\
\hline $\begin{array}{l}\text { Blinding of outcome as- } \\
\text { sessment (detection bias) } \\
\text { All outcomes }\end{array}$ & High risk & Open-label trial \\
\hline $\begin{array}{l}\text { Incomplete outcome data } \\
\text { (attrition bias) } \\
\text { All outcomes }\end{array}$ & Low risk & All 61 randomised infants were included in the analysis \\
\hline $\begin{array}{l}\text { Selective reporting (re- } \\
\text { porting bias) }\end{array}$ & Low risk & Study protocol had been published. All proposed outcomes were reported \\
\hline Other bias & Low risk & Nil \\
\hline
\end{tabular}

CVL: central venous line.

NEC: necrotising enterocolitis.

Routine monitoring of gastric residual for prevention of necrotising enterocolitis in preterm infants (Review) 
$\mathrm{RCT}$ : randomised controlled trial.

TPN: total parenteral nutrition.

Characteristics of excluded studies [ordered by study ID]

\begin{tabular}{ll}
\hline Study & Reason for exclusion \\
\hline Bertino 2009 & This is a case-control study \\
\hline Cobb 2004 & This is a case-control study \\
\hline Malhotra 1992 & This is a prospective observational study \\
\hline Mihatsch 2002 & This is a prospective observational study \\
\hline Riskin 2017 & This is a case-control study \\
\hline
\end{tabular}

Characteristics of ongoing studies [ordered by study ID]

Aljariry 2017

\begin{tabular}{ll}
\hline Trial name or title & Routine gastric residual aspiration in preterm infants and the effect on reaching full feed \\
\hline Methods & RCT \\
\hline Participants & Infants $\leq 32$ weeks' gestational age \\
\hline Interventions & Routine gastric aspiration group: routine prefeed aspiration of gastric residuals every 6 hours \\
& No aspiration group: no routine gastric residual aspiration \\
\hline Outcomes & $\begin{array}{l}\text { Primary outcome: time taken to reach full enteral feeds }(120 \mathrm{~mL} / \mathrm{kg} / \mathrm{d}) \\
\text { Secondary outcomes: time taken to reach } 150 \mathrm{~mL} / \mathrm{kg} / \mathrm{d}, \mathrm{duration} \text { of TPN, duration of CVL usage, in- } \\
\text { cidence of sepsis, incidence of NEC, and weight gain }(\mathrm{g} / \mathrm{kg} / \mathrm{d})\end{array}$ \\
\hline Starting date & December 2015 \\
\hline Contact information & www.isrctn.com/ISRCTN98322846 \\
\hline Notes & Study completion estimated for December 2018 \\
\hline
\end{tabular}

\section{GRASS 2017}

\begin{tabular}{ll}
\hline Trial name or title & $\begin{array}{l}\text { Does routine assessment of gastric residuals in preterm neonates influence time taken to reach full } \\
\text { enteral feeding? (GRASS) }\end{array}$ \\
\hline Methods & RCT \\
\hline Participants & Infants born at 26 to 30 weeks' gestational age and birth weight $<1500 \mathrm{~g}$ \\
\hline Interventions & $\begin{array}{l}\text { Intervention: no routine aspiration of prefeed gastric residuals. Opening of the nasogastric tube } \\
\text { once every } 6 \text { hours to relieve possible backflow of gastric content will be allowed } \\
\text { Comparison: routine monitoring of gastric residuals before each feed }\end{array}$ \\
\hline
\end{tabular}


GRASS 2017 (Continued)

Outcomes

Primary outcome: time taken to achieve full enteral feeding $(100 \mathrm{~mL} / \mathrm{kg} / \mathrm{d})$

Secondary outcomes: episodes of withholding of enteral feeding, duration of TPN, duration of CVL usage, hypoglycaemia, late-onset sepsis, NEC, spontaneous intestinal perforation, bronchopulmonary dysplasia, intraventricular haemorrhage, retinopathy of prematurity, and neurodevelopment at 24 months

\begin{tabular}{ll}
\hline Starting date & October 2017 \\
\hline Contact information & www.clinicaltrials.gov/ct2/show/NCT03111329 \\
\hline Notes & Study completion estimated for December 2018 \\
\hline
\end{tabular}

Parker 2013

\begin{tabular}{ll}
\hline Trial name or title & Aspiration of residual gastric contents \\
\hline Methods & RCT \\
\hline Participants & $\begin{array}{l}\text { Infants } \leq 32 \text { weeks' gestational age and birth weight } \leq 1250 \text { g receiving some enteral feeds by } 72 \\
\text { hours of age }\end{array}$ \\
\hline Interventions & Intervention: no routine monitoring of gastric residuals \\
& Comparison: routine monitoring of gastric residuals before each feed \\
\hline Outcomes & $\begin{array}{l}\text { Primary outcome: } 24-h o u r \text { enteral feeding intake in mL/kg } \\
\text { Secondary outcomes: time to reach full feeds, duration of TPN, duration of CVL usage, NEC, aspira- } \\
\text { tion pneumonia, ventilator-associated pneumonia, and length of hospital stay }\end{array}$ \\
\hline Starting date & May 2013 \\
\hline Contact information & www.clinicaltrials.gov/ct2/show/NCT01863043 \\
\hline Notes & Study completion estimated for February 2019 \\
\hline
\end{tabular}

CVL: central venous line.

NEC: necrotising enterocolitis.

RCT: randomised controlled trial.

TPN: total parenteral nutrition.

\section{DATA AND ANALYSES}

\section{Comparison 1. Routine monitoring of gastric residuals versus no monitoring of gastric residuals}

\begin{tabular}{lllll}
\hline Outcome or subgroup title & No. of studies & $\begin{array}{l}\text { No. of partici- } \\
\text { pants }\end{array}$ & Statistical method & Effect size \\
\hline $\begin{array}{l}1 \text { Number of infants with NEC stage 2 } \\
\text { or } 3\end{array}$ & 2 & 141 & $\begin{array}{l}\text { Risk Ratio (M-H, Fixed, 95\% } \\
\text { Cl) }\end{array}$ & 3.07 [0.50, 18.77] \\
\hline 2 Time to reach full enteral feeds & 2 & 141 & $\begin{array}{l}\text { Mean Difference (IV, Fixed, } \\
95 \% \text { CI) }\end{array}$ & 3.92 [2.06, 5.77] \\
\hline \hline
\end{tabular}




\begin{tabular}{|c|c|c|c|c|}
\hline Outcome or subgroup title & No. of studies & $\begin{array}{l}\text { No. of partici- } \\
\text { pants }\end{array}$ & Statistical method & Effect size \\
\hline 3 Number of infants with surgical NEC & 1 & 80 & $\begin{array}{l}\text { Risk Ratio (M-H, Fixed, 95\% } \\
\mathrm{Cl})\end{array}$ & $1.25[0.36,4.32]$ \\
\hline 4 Time to regain birth weight (days) & 1 & 80 & $\begin{array}{l}\text { Mean Difference (IV, Fixed, } \\
95 \% \mathrm{CI} \text { ) }\end{array}$ & $1.70[0.01,3.39]$ \\
\hline $\begin{array}{l}5 \text { Number of infants with extrauterine } \\
\text { growth restriction at discharge }\end{array}$ & 1 & 80 & $\begin{array}{l}\text { Risk Ratio (M-H, Fixed, 95\% } \\
\mathrm{Cl} \text { ) }\end{array}$ & $0.89[0.75,1.05]$ \\
\hline $\begin{array}{l}6 \text { Number of infants with episodes } \\
\text { of interruption of feeds (lasting } \geq 12 \\
\text { hours) }\end{array}$ & 2 & 141 & $\begin{array}{l}\text { Risk Ratio (M-H, Fixed, 95\% } \\
\mathrm{Cl} \text { ) }\end{array}$ & $2.07[1.39,3.07]$ \\
\hline 7 Number of TPN days & 2 & 141 & $\begin{array}{l}\text { Mean Difference (IV, Fixed, } \\
95 \% \mathrm{CI} \text { ) }\end{array}$ & $3.29[1.66,4.92]$ \\
\hline $\begin{array}{l}8 \text { Number of infants with parenteral } \\
\text { nutrition-associated liver disease }\end{array}$ & 1 & 61 & $\begin{array}{l}\text { Risk Ratio (M-H, Fixed, 95\% } \\
\mathrm{Cl} \text { ) }\end{array}$ & $1.03[0.28,3.76]$ \\
\hline 9 Number of days of CVL usage & 1 & 61 & $\begin{array}{l}\text { Mean Difference (IV, Fixed, } \\
95 \% \mathrm{Cl})\end{array}$ & $5.70[-1.99,13.39]$ \\
\hline $\begin{array}{l}10 \text { Number of infants with invasive in- } \\
\text { fection }\end{array}$ & 2 & 141 & $\begin{array}{l}\text { Risk Ratio (M-H, Fixed, 95\% } \\
\mathrm{Cl} \text { ) }\end{array}$ & $1.46[0.85,2.52]$ \\
\hline $\begin{array}{l}11 \text { All-cause mortality before dis- } \\
\text { charge }\end{array}$ & 1 & 80 & $\begin{array}{l}\text { Risk Ratio (M-H, Fixed, 95\% } \\
\mathrm{Cl} \text { ) }\end{array}$ & $1.25[0.36,4.32]$ \\
\hline 12 Duration of hospital stay (days) & 1 & 80 & $\begin{array}{l}\text { Mean Difference (IV, Fixed, } \\
95 \% \mathrm{CI} \text { ) }\end{array}$ & $3.20[-4.45,10.85]$ \\
\hline
\end{tabular}

Analysis 1.1. Comparison 1 Routine monitoring of gastric residuals versus no monitoring of gastric residuals, Outcome 1 Number of infants with NEC stage 2 or 3.

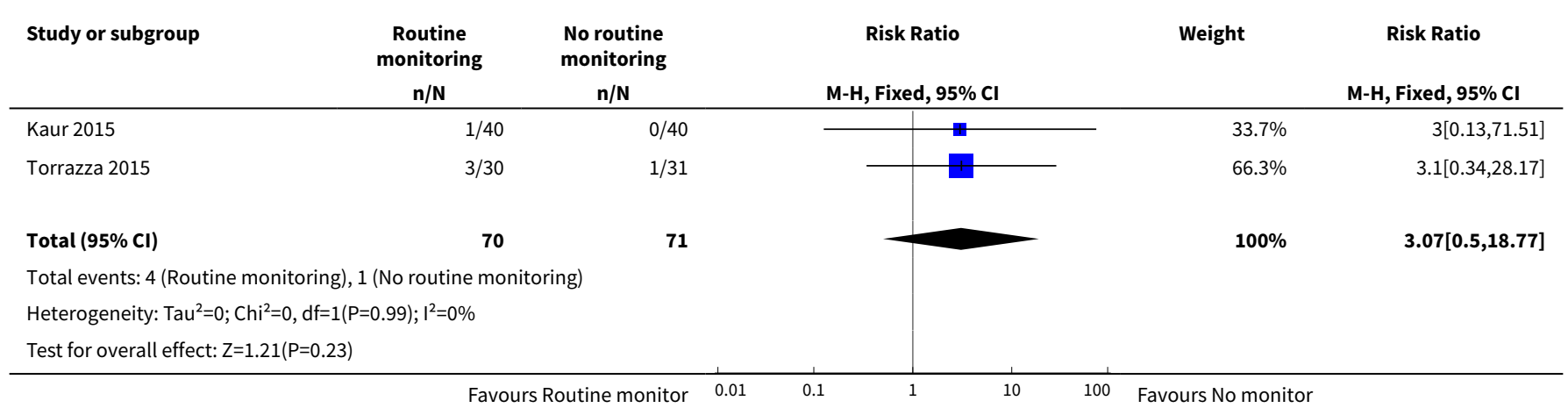


Analysis 1.2. Comparison 1 Routine monitoring of gastric residuals versus no monitoring of gastric residuals, Outcome 2 Time to reach full enteral feeds.

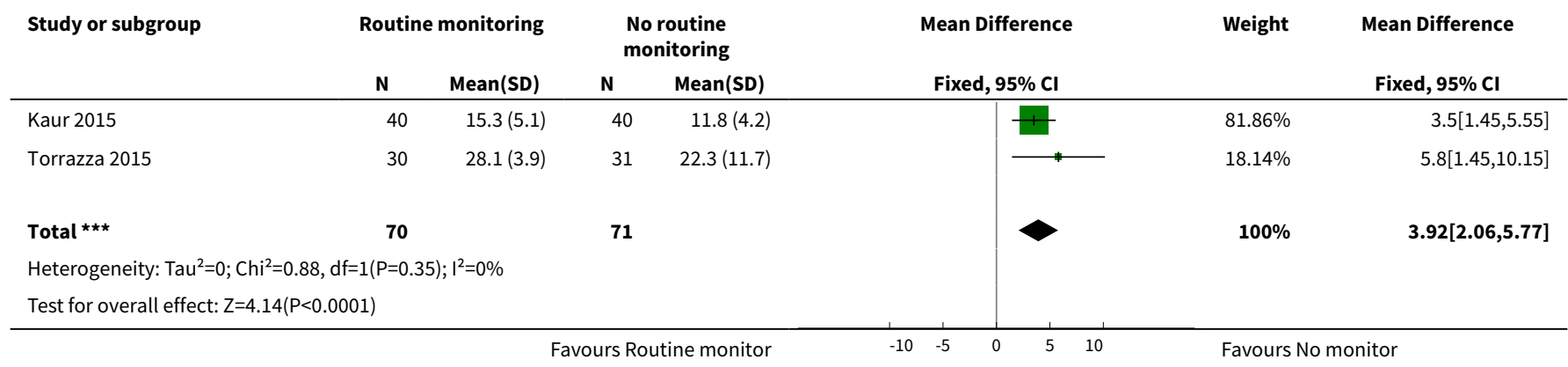

Analysis 1.3. Comparison 1 Routine monitoring of gastric residuals versus no monitoring of gastric residuals, Outcome 3 Number of infants with surgical NEC.

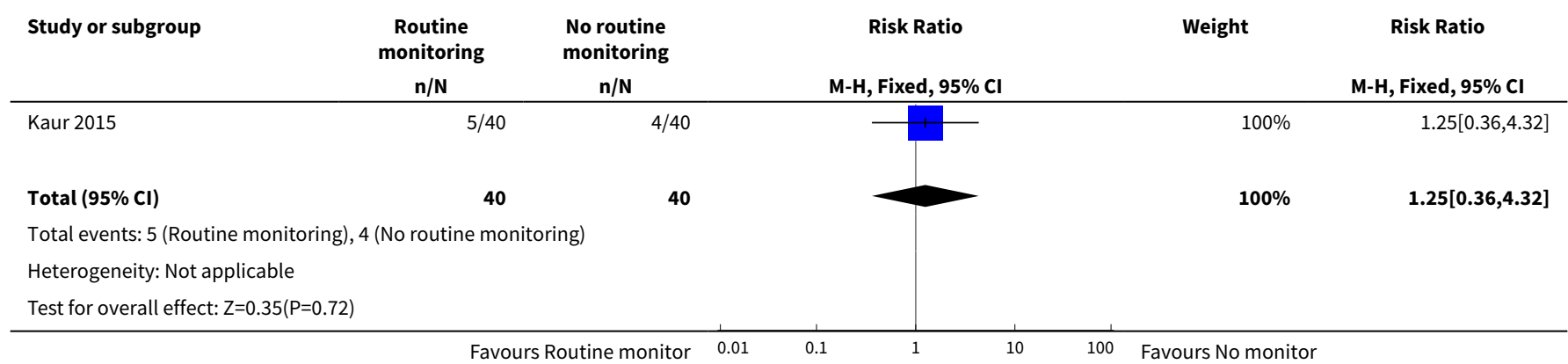

Analysis 1.4. Comparison 1 Routine monitoring of gastric residuals versus no monitoring of gastric residuals, Outcome 4 Time to regain birth weight (days).

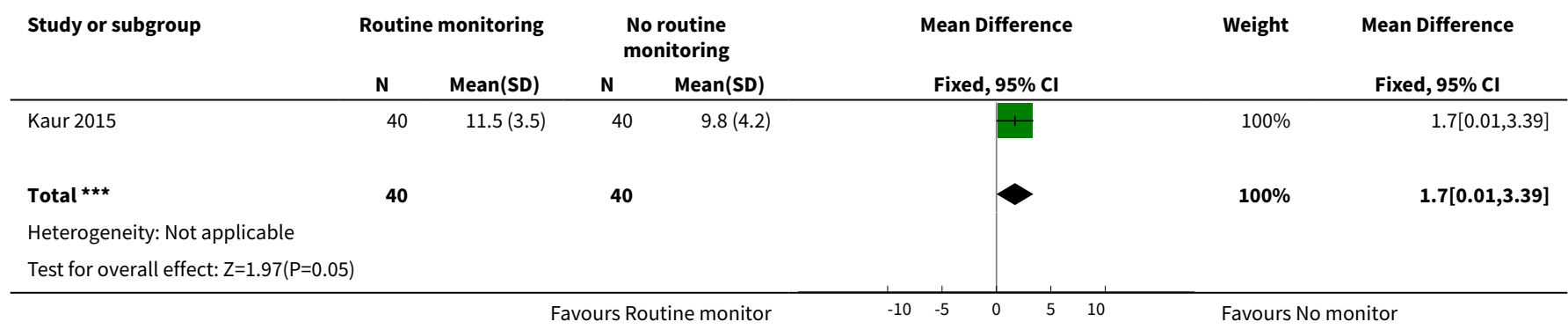

Analysis 1.5. Comparison 1 Routine monitoring of gastric residuals versus no monitoring of gastric residuals, Outcome 5 Number of infants with extrauterine growth restriction at discharge.

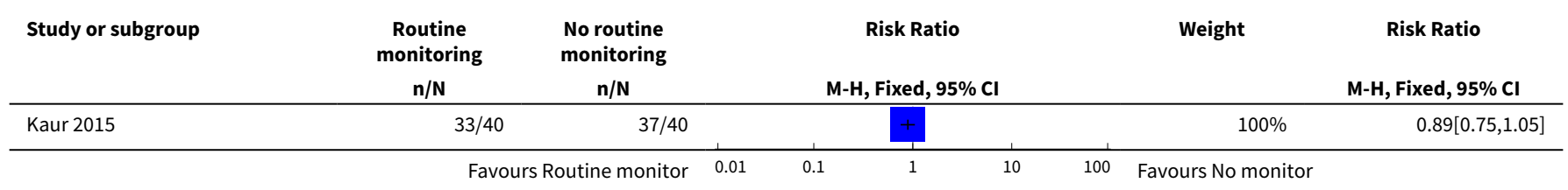




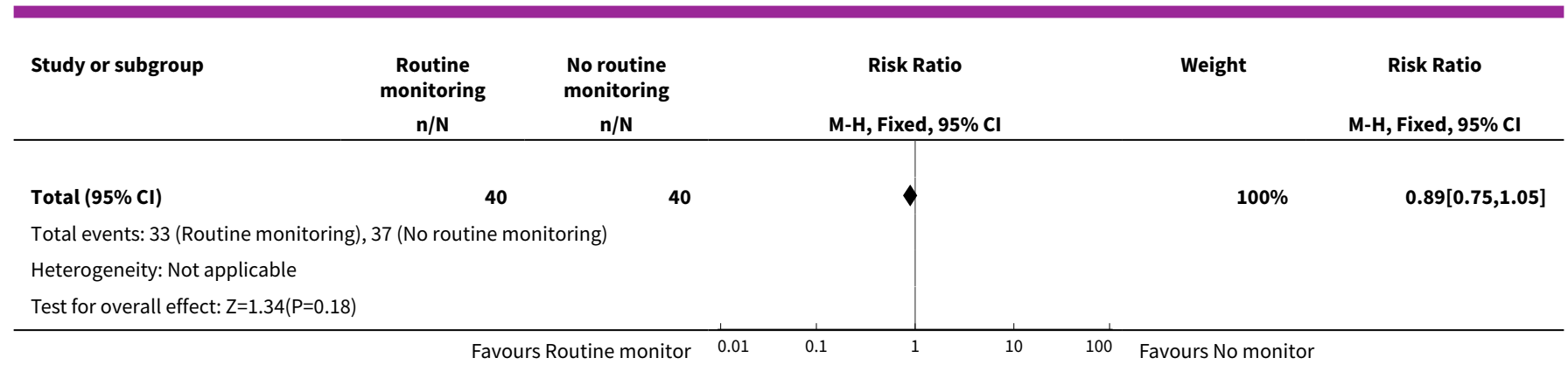

Analysis 1.6. Comparison 1 Routine monitoring of gastric residuals versus no monitoring of gastric residuals, Outcome 6 Number of infants with episodes of interruption of feeds (lasting $\geq 12$ hours).

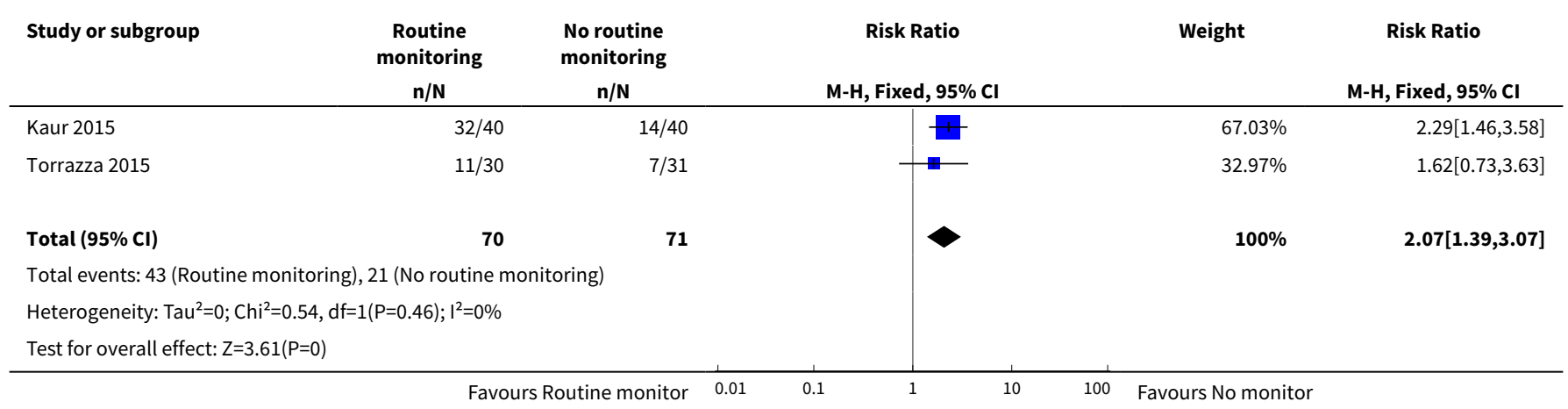

Analysis 1.7. Comparison 1 Routine monitoring of gastric residuals versus no monitoring of gastric residuals, Outcome 7 Number of TPN days.

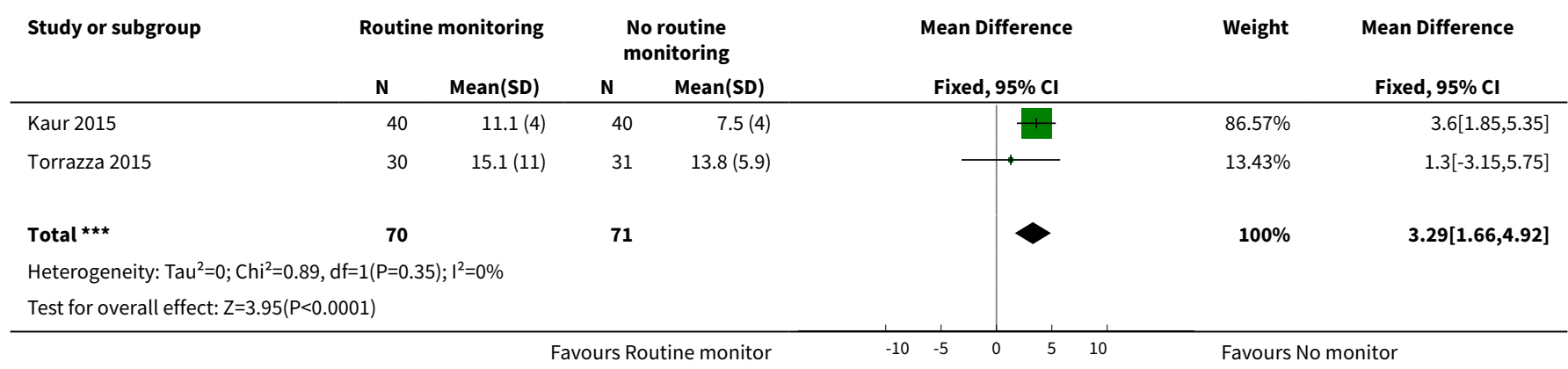

Analysis 1.8. Comparison 1 Routine monitoring of gastric residuals versus no monitoring of gastric residuals, Outcome 8 Number of infants with parenteral nutrition-associated liver disease.

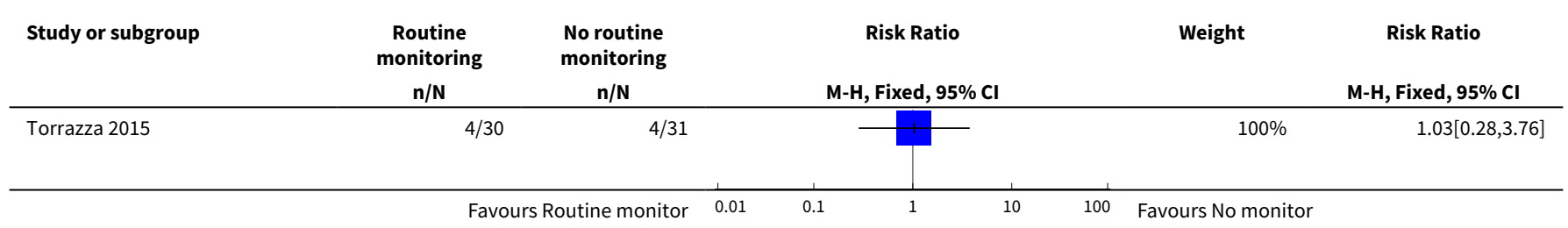




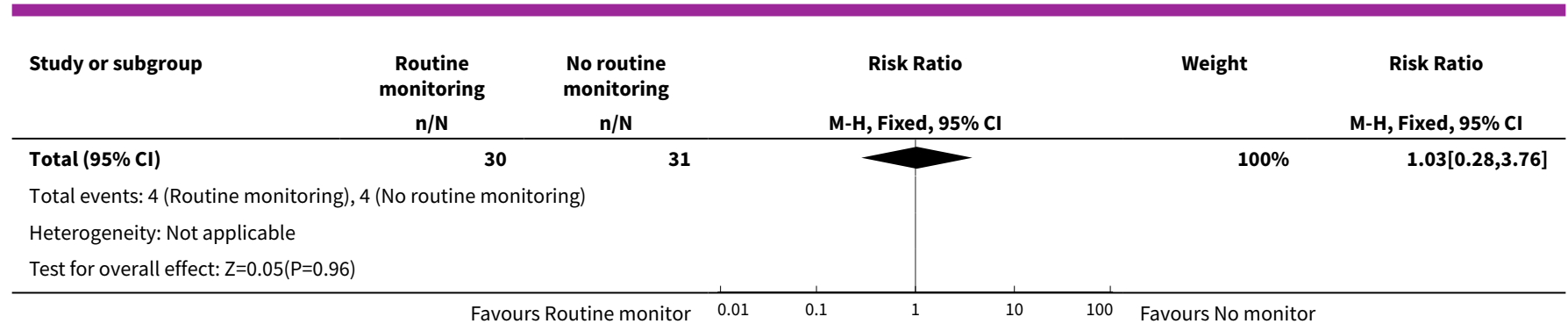

Analysis 1.9. Comparison 1 Routine monitoring of gastric residuals versus no monitoring of gastric residuals, Outcome 9 Number of days of CVL usage.

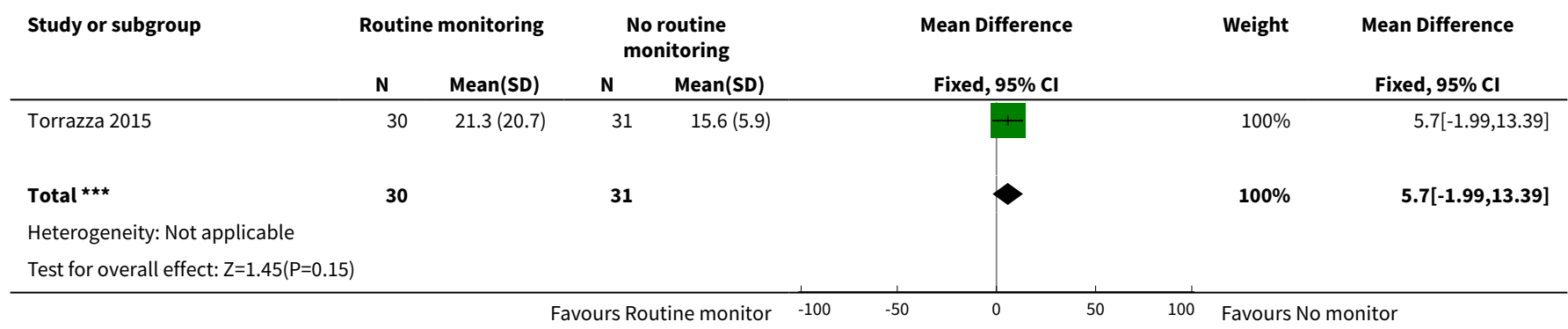

Analysis 1.10. Comparison 1 Routine monitoring of gastric residuals versus no monitoring of gastric residuals, Outcome 10 Number of infants with invasive infection.

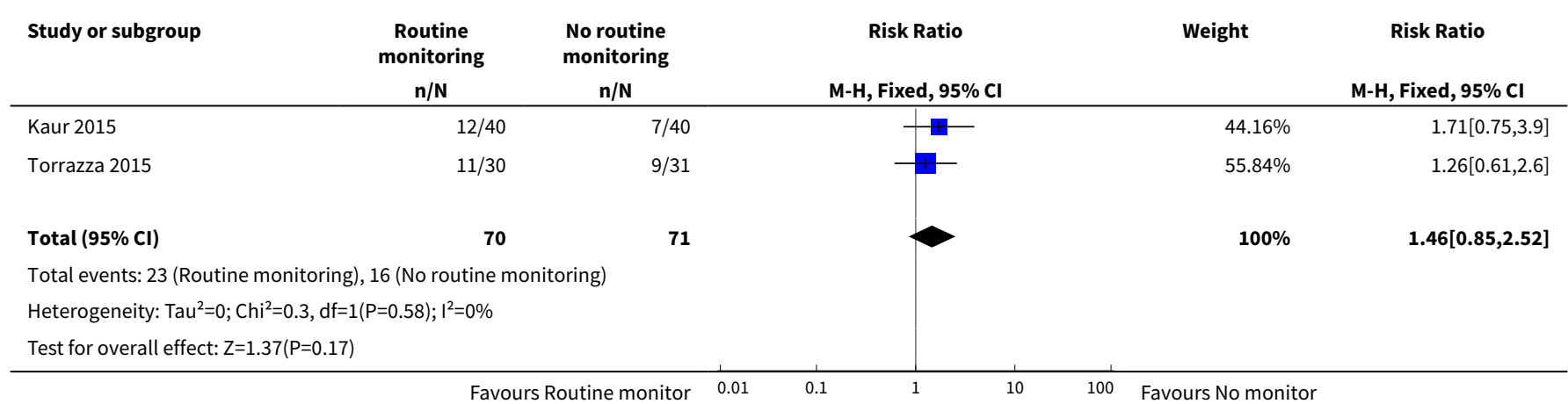

Analysis 1.11. Comparison 1 Routine monitoring of gastric residuals versus no monitoring of gastric residuals, Outcome 11 All-cause mortality before discharge.

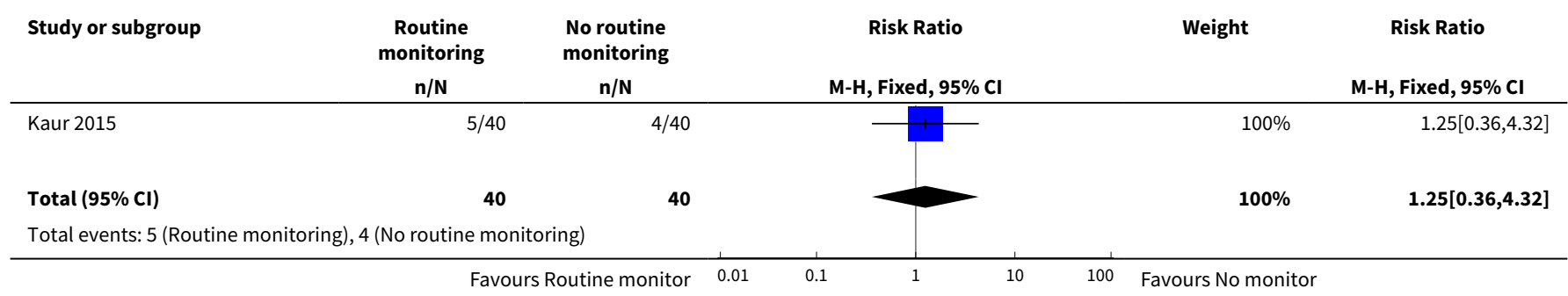




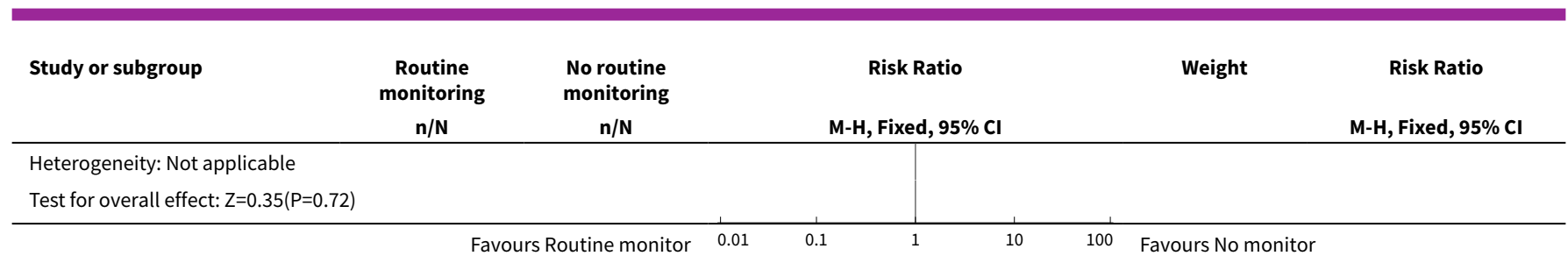

Analysis 1.12. Comparison 1 Routine monitoring of gastric residuals versus no
monitoring of gastric residuals, Outcome 12 Duration of hospital stay (days).

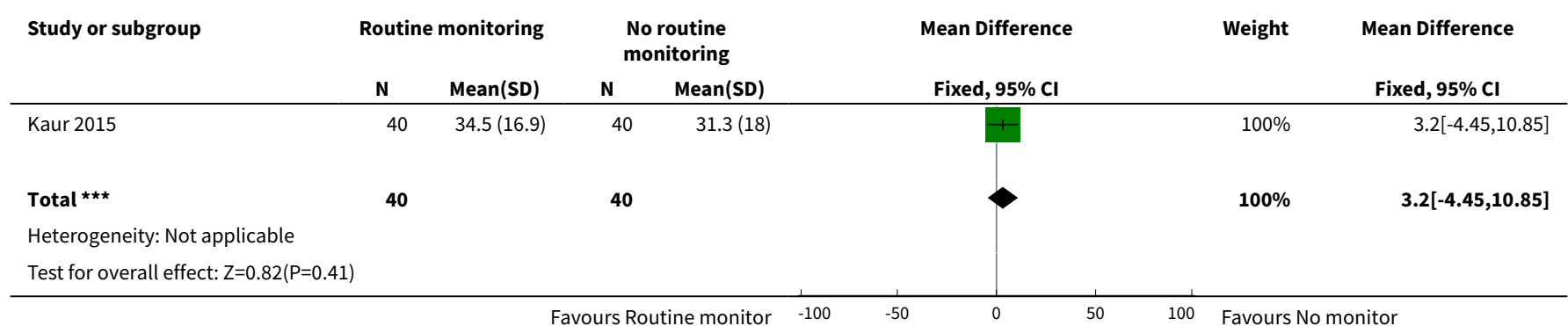

Comparison 2. Using two different criteria of gastric residual for feed interruption while monitoring gastric residual

\begin{tabular}{|c|c|c|c|c|}
\hline Outcome or subgroup title & No. of studies & $\begin{array}{l}\text { No. of partici- } \\
\text { pants }\end{array}$ & Statistical method & Effect size \\
\hline $\begin{array}{l}1 \text { Number of infants with NEC stage } 2 \\
\text { or } 3\end{array}$ & 1 & 87 & $\begin{array}{l}\text { Risk Ratio (M-H, Fixed, 95\% } \\
\mathrm{Cl})\end{array}$ & $5.35[0.26,108.27]$ \\
\hline 2 TIme to reach full enteral feeds & 1 & 87 & $\begin{array}{l}\text { Mean Difference (IV, Fixed, } \\
95 \% \mathrm{Cl} \text { ) }\end{array}$ & $-0.10[-0.91,0.71]$ \\
\hline 3 Number of infants with surgical NEC & 1 & 87 & $\begin{array}{l}\text { Risk Ratio (M-H, Fixed, 95\% } \\
\mathrm{Cl})\end{array}$ & $5.35[0.26,108.27]$ \\
\hline 4 Time to regain birth weight (days) & 1 & 87 & $\begin{array}{l}\text { Mean Difference (IV, Fixed, } \\
95 \% \mathrm{Cl} \text { ) }\end{array}$ & $1.0[-0.37,2.37]$ \\
\hline $\begin{array}{l}5 \text { Number of infants with extrauterine } \\
\text { growth restriction at discharge }\end{array}$ & 1 & 87 & $\begin{array}{l}\text { Risk Ratio (M-H, Fixed, 95\% } \\
\text { Cl) }\end{array}$ & $0.54[0.14,2.01]$ \\
\hline $\begin{array}{l}6 \text { Number of infants with episodes } \\
\text { of interruption of feeds (lasting } \geq 12 \\
\text { hours) }\end{array}$ & 1 & 87 & $\begin{array}{l}\text { Risk Ratio (M-H, Fixed, 95\% } \\
\mathrm{Cl} \text { ) }\end{array}$ & $3.21[0.13,76.67]$ \\
\hline 7 Number of TPN days & 1 & 87 & $\begin{array}{l}\text { Mean Difference (IV, Fixed, } \\
95 \% \mathrm{CI} \text { ) }\end{array}$ & $0.80[-0.78,2.38]$ \\
\hline $\begin{array}{l}8 \text { Number of infants with Invasive In- } \\
\text { fection }\end{array}$ & 1 & 87 & $\begin{array}{l}\text { Risk Ratio (M-H, Fixed, 95\% } \\
\mathrm{Cl})\end{array}$ & $5.35[0.26,108.27]$ \\
\hline 9 All-cause mortality before discharge & 1 & 87 & $\begin{array}{l}\text { Risk Ratio (M-H, Fixed, 95\% } \\
\mathrm{Cl})\end{array}$ & $3.21[0.13,76.67]$ \\
\hline
\end{tabular}


Analysis 2.1. Comparison 2 Using two different criteria of gastric residual for feed interruption while monitoring gastric residual, Outcome 1 Number of infants with NEC stage 2 or 3.

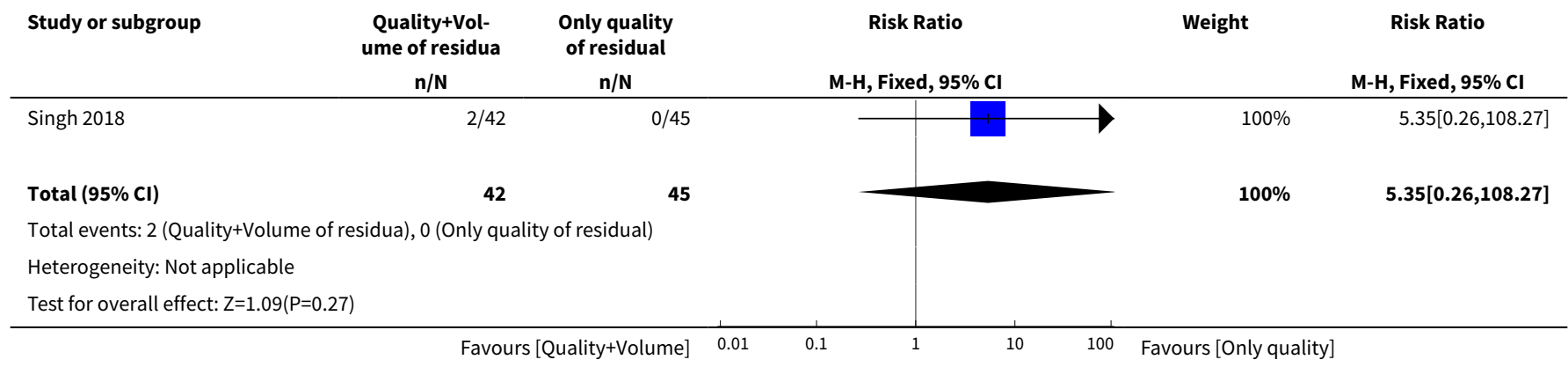

Analysis 2.2. Comparison 2 Using two different criteria of gastric residual for feed interruption while monitoring gastric residual, Outcome 2 TIme to reach full enteral feeds.

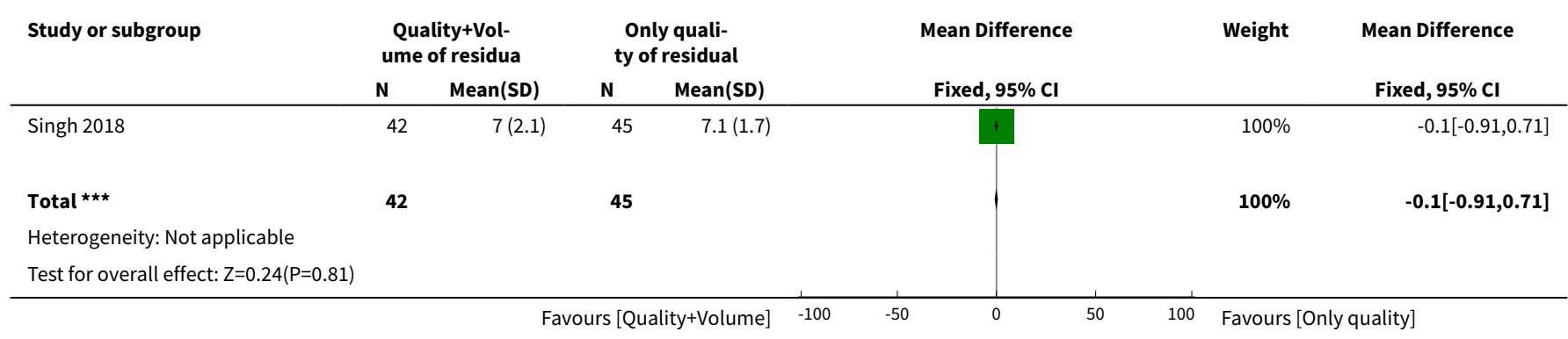

Analysis 2.3. Comparison 2 Using two different criteria of gastric residual for feed interruption while monitoring gastric residual, Outcome 3 Number of infants with surgical NEC.

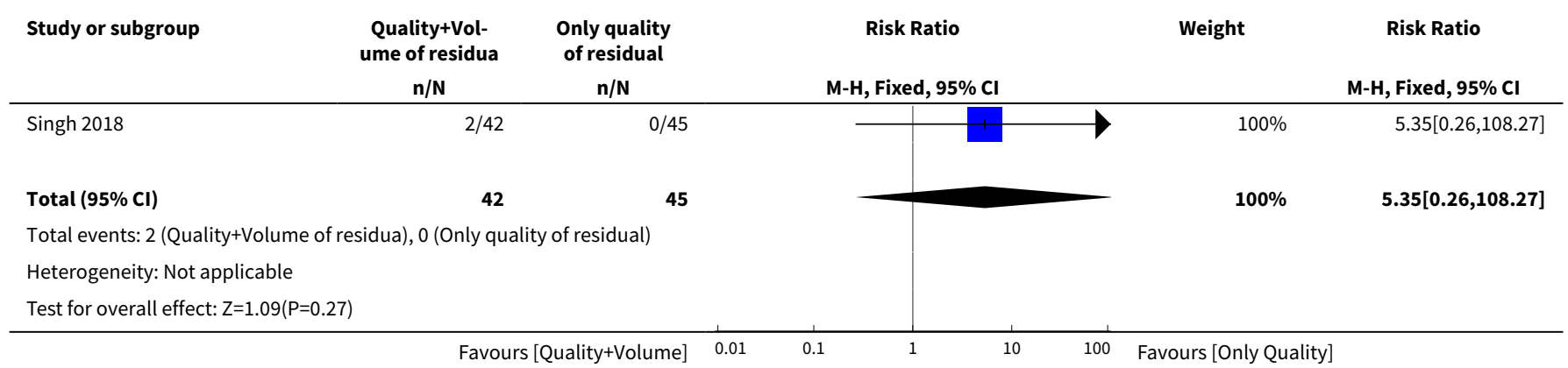


Analysis 2.4. Comparison 2 Using two different criteria of gastric residual for feed interruption while monitoring gastric residual, Outcome 4 Time to regain birth weight (days).

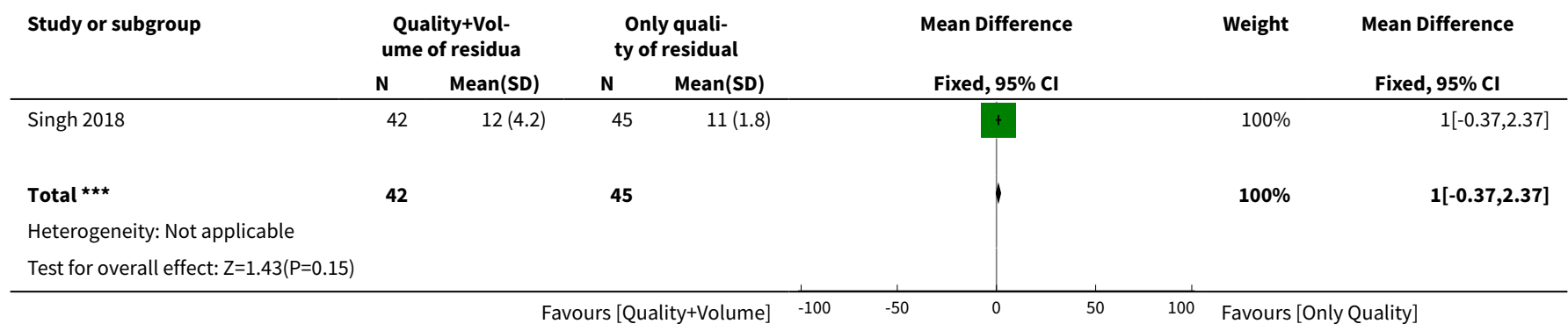

Analysis 2.5. Comparison 2 Using two different criteria of gastric residual for feed interruption while monitoring gastric residual, Outcome 5 Number of infants with extrauterine growth restriction at discharge.

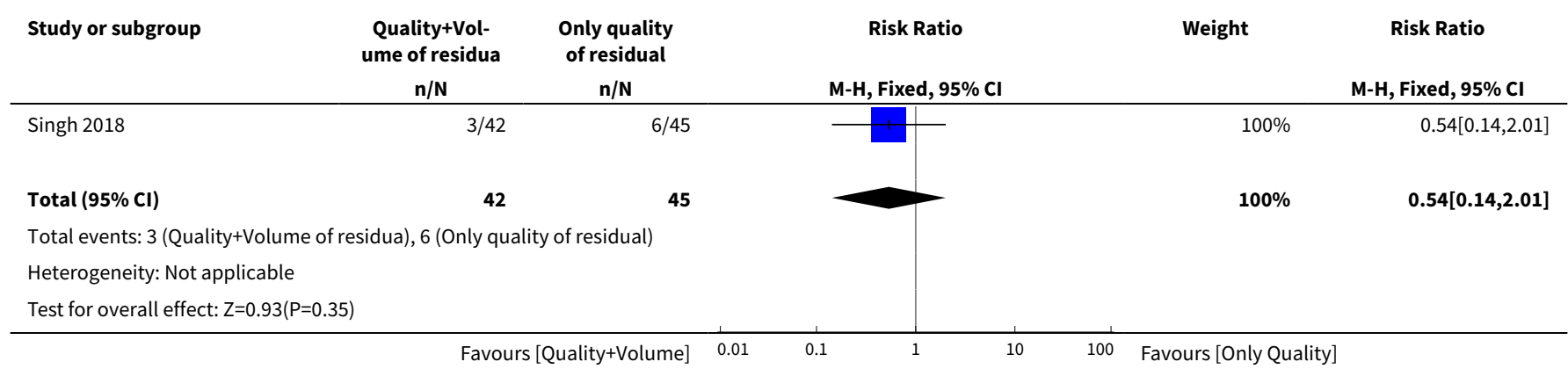

Analysis 2.6. Comparison 2 Using two different criteria of gastric residual for feed interruption while monitoring gastric residual, Outcome 6 Number of infants with episodes of interruption of feeds (lasting $\geq 12$ hours).

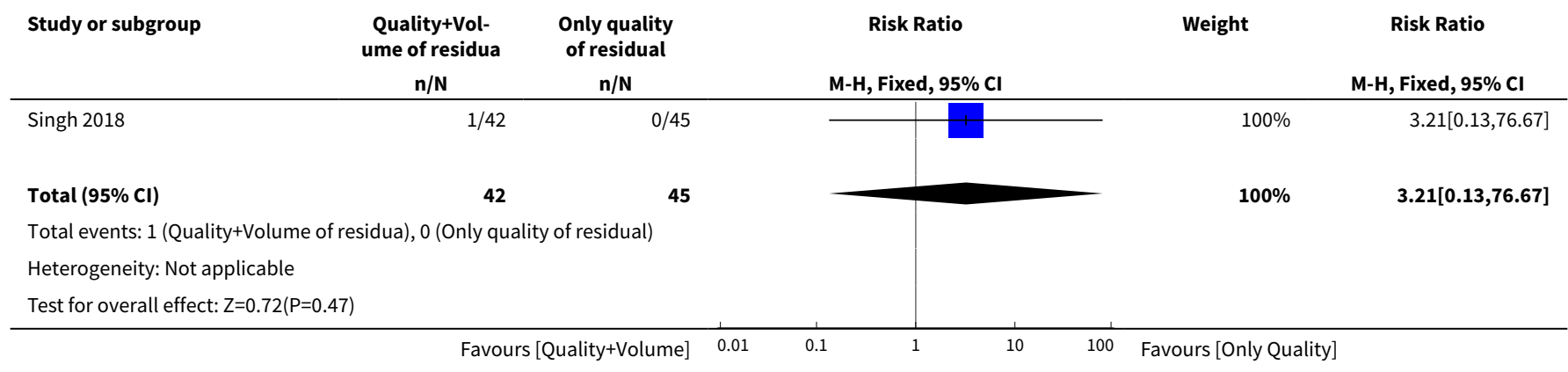

Analysis 2.7. Comparison 2 Using two different criteria of gastric residual for feed interruption while monitoring gastric residual, Outcome 7 Number of TPN days.

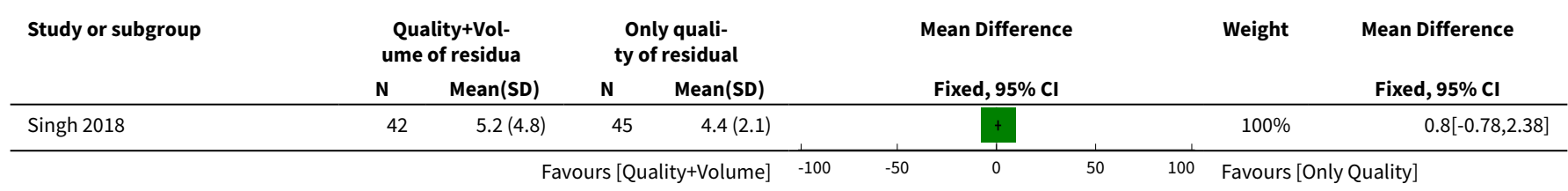




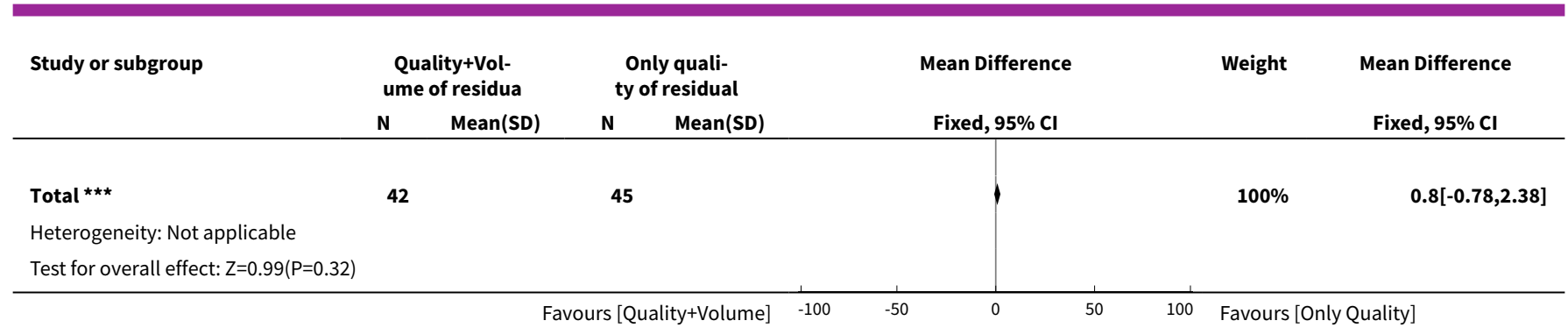

\section{Analysis 2.8. Comparison 2 Using two different criteria of gastric residual for feed interruption while monitoring gastric residual, Outcome 8 Number of infants with Invasive Infection.}

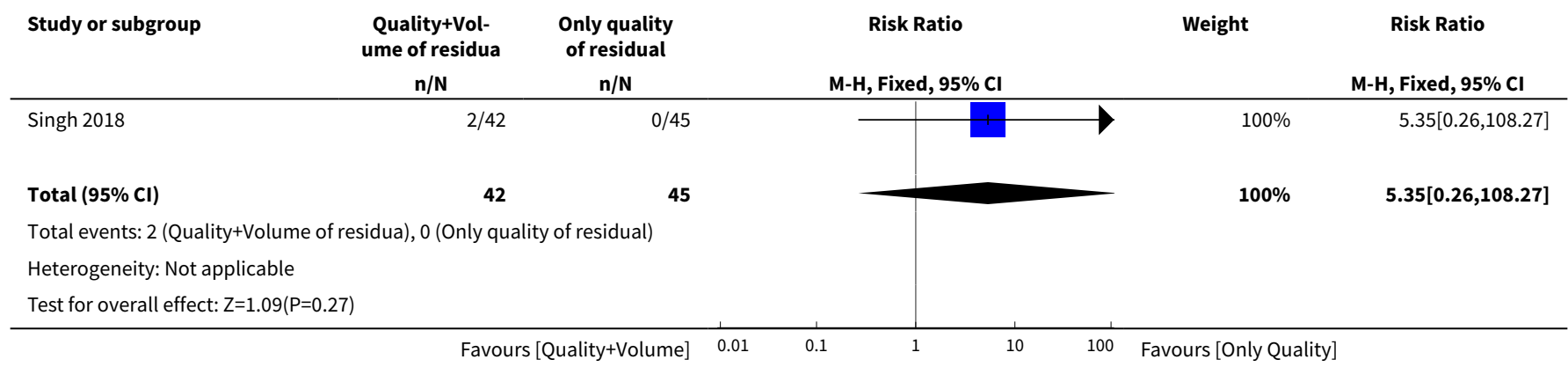

Analysis 2.9. Comparison 2 Using two different criteria of gastric residual for feed interruption while monitoring gastric residual, Outcome 9 All-cause mortality before discharge.

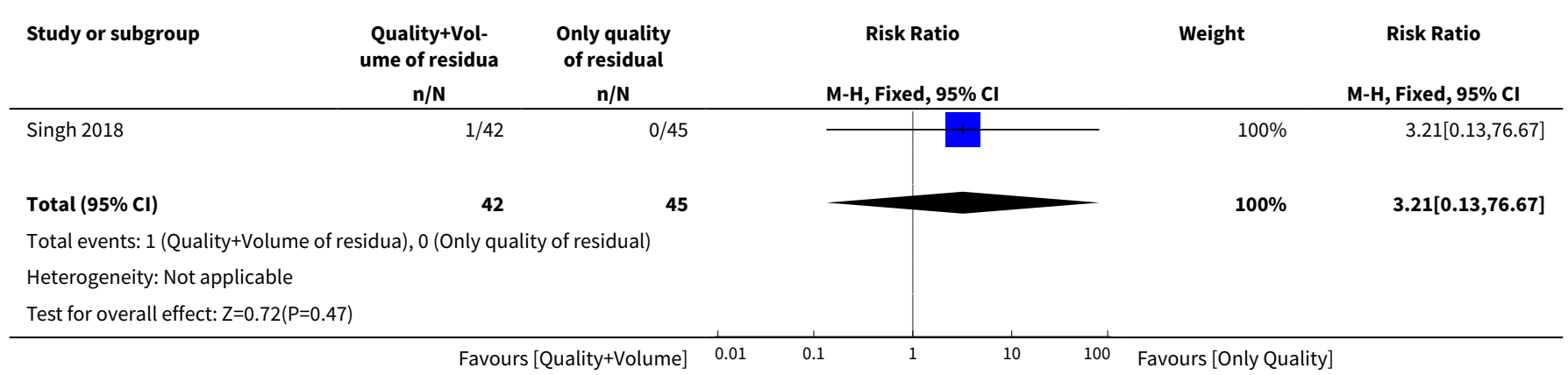

\section{AP PEN DICES}

\section{Appendix 1. Cochrane Neonatal standard search strategy}

PubMed: ((infant, newborn[MeSH] OR newborn OR neonate OR neonatal OR premature OR low birth weight OR VLBW OR LBW or infan* or neonat $^{\star}$ ) AND (randomised controlled trial [pt] OR controlled clinical trial [pt] OR randomised [tiab] OR placebo [tiab] OR drug therapy [sh] OR randomly [tiab] OR trial [tiab] OR groups [tiab]) NOT (animals [mh] NOT humans [mh]))

Embase: ((exp infant) OR (infan* OR newborn or neonat* OR premature or very low birth weight or low birth weight or VLBW or LBW).mp AND (human not animal) AND (randomised controlled trial or controlled clinical trial or randomised or placebo or clinical trials as topic or randomly or trial or clinical trial).mp 
CINAHL: (infan* OR newborn OR neonat* OR premature OR low birth weight OR VLBW OR LBW) AND (randomised controlled trial OR controlled clinical trial OR randomised OR placebo OR clinical trials as topic OR randomly OR trial OR PT clinical trial)

CRS Web: (infan* or newborn or neonat* or premature or preterm or very low birth weight or low birth weight or VLBW or LBW)

\section{Appendix 2. Risk of bias tool}

\section{Sequence generation (checking for possible selection bias). Was the allocation sequence adequately generated?}

For each included study, we categorised the method used to generate the allocation sequence as:

- low risk (any truly random process, e.g. random number table; computer random number generator);

- high risk (any non-random process, e.g. odd or even date of birth; hospital or clinic record number); or

- unclear risk.

\section{Allocation concealment (checking for possible selection bias). Was allocation adequately concealed?}

For each included study, we categorised the method used to conceal the allocation sequence as:

- low risk (e.g. telephone or central randomisation; consecutively numbered sealed opaque envelopes);

- high risk (open random allocation; unsealed or non-opaque envelopes, alternation; date of birth); or

- unclear risk.

3. Blinding of participants and personnel (checking for possible performance bias). Was knowledge of the allocated intervention adequately prevented during the study?

For each included study, we categorised the methods used to blind study participants and personnel from knowledge of which intervention a participant received. Blinding was assessed separately for different outcomes or class of outcomes. We categorised the methods as:

- low risk, high risk, or unclear risk for participants; and

- low risk, high risk, or unclear risk for personnel.

4. Blinding of outcome assessment (checking for possible detection bias). Was knowledge of the allocated intervention adequately prevented at the time of outcome assessment?

For each included study, we categorised the methods used to blind outcome assessment. Blinding was assessed separately for different outcomes or classes of outcomes. We categorised the methods as:

- low risk for outcome assessors;

- high risk for outcome assessors; or

- unclear risk for outcome assessors.

5. Incomplete outcome data (checking for possible attrition bias through withdrawals, dropouts, protocol deviations). Were incomplete outcome data adequately addressed?

For each included study and for each outcome, we described the completeness of data including attrition and exclusions from the analysis. We noted whether attrition and exclusions were reported, numbers included in the analysis at each stage (compared with the total randomised participants), reasons for attrition or exclusion where reported, and whether missing data were balanced across groups or were related to outcomes. Where sufficient information was reported or supplied by trial authors, we re-included missing data in the analyses. We categorised the methods as:

- low risk (<20\% missing data);

- high risk ( $\geq 20 \%$ missing data); or

- unclear risk.

\section{Selective reporting bias. Are reports of the study free of suggestion of selective outcome reporting?}

For each included study, we described how we investigated the possibility of selective outcome reporting bias and what we found. For studies in which study protocols were published in advance, we compared prespecified outcomes versus outcomes eventually reported in 
the published results. If the study protocol was not published in advance, we contacted study authors to gain access to the study protocol. We assessed the methods as:

- low risk (where it is clear that all of the study's prespecified outcomes and all expected outcomes of interest to the review have been reported);

- high risk (where not all of the study's prespecified outcomes have been reported; one or more reported primary outcomes were not prespecified outcomes of interest and are reported incompletely and so cannot be used; study fails to include results of a key outcome that would have been expected to have been reported); or

- unclear risk.

\section{Other sources of bias. Was the study apparently free of other problems that could put it at a high risk of bias?}

For each included study, we described any important concerns we had about other possible sources of bias (e.g. whether there was a potential source of bias related to the specific study design, whether the trial was stopped early due to some data-dependent process). We assessed whether each study was free of other problems that could put it at risk of bias as:

- low risk;

- high risk; or

- unclear risk.

If needed, we explored the impact of the level of bias through undertaking sensitivity analyses.

\section{CONTRIBUTIONS OF AUTHORS}

TA and ST developed the protocol. TA and ST screened search outputs, assessed study eligibility, and extracted and synthesised data. TA and ST assessed risk of bias across key domains and undertook GRADE assessment. All review authors revised the final review.

\section{DECLARATIONS OF INTEREST}

TA has no interests to declare.

ST has no interests to declare.

BN has no interests to declare.

\section{SOURCES OF SUPPORT}

\section{Internal sources}

- Sri Ramachandra Medical College and Research Institute, Chennai, India.

- Christian Medical College, Vellore, India.

\section{External sources}

- National Institute for Health Research, UK.

Editorial support for Cochrane Neonatal has been funded with funds from a UK National Institute of Health Research (NIHR) Cochrane Programme Grant (16/114/03). The views expressed in this publication are those of the authors and not necessarily those of the National Health Service, the NIHR or the UK Department of Health.

- Vermont Oxford Network, USA.

Cochrane Neonatal Reviews are produced with support from Vermont Oxford Network, a worldwide collaboration of health professionals dedicated to providing evidence-based care of the highest quality for newborn infants and their families.

\section{DIFFERENCES BETWEEN PROTOCOLAND REVIEW}

For the outcome of feed interruption, while we planned to analyse the number of episodes of feed interruption lasting $\geq 12$ hours in each group, trialists of the included trials reported the number of infants with episodes of feed interruption lasting $\geq 12$ hours.

We did not pre-specify the outcomes for the Summary of findings table in the protocol. 